\title{
Signal Processing by Generalized Receiver Using DS-CDMA with STBC Technology in Multidimensional Systems over Fading Channels: Capacity and Error Probability
}

\author{
VYACHESLAV TUZLUKOV \\ Department of Technical Maintenance of Aviation and Radio Electronic Equipment \\ Belarusian State Aviation Academy \\ 77, Uborevicha Str., 220096 Minsk \\ BELARUS
}

\begin{abstract}
The capacity and error probability of orthogonal space-time block codes (STBCs) are considered for the pulse-amplitude modulation (PAM), phase shift keying (PSK), quadrature-amplitude modulation (QAM) in fading channels. The suggested approach is based on employment of the generalized approach to signal processing in noise over the equivalent scalar additive white Gaussian noise channel with a channel gain proportional to the Frobenius norm of the matrix channel for the STBC. Using this effective channel, the capacity and probability of error expressions are derived for the PSK, PAM, and QAM modulation with space-time block coding. The Rayleigh, Rician and Nakagami fading channels are considered. As an application, these results are extended to obtain the capacity and probability of error for a multiuser direct sequence code-division multiple-access (CDMA) system constructed on the basis of the generalized approach to signal processing in noise employing the space-time block coding.
\end{abstract}

Key-Words: - Generalized receiver, code-division multiple-access (CDMA), fading channels, phase shift keying, pulse amplitude modulation, quadrature amplitude modulation, Rayleigh channel, Rician channel.

Received: January 6, 2020. Revised: August 8, 2020. Accepted: September 9, 2020. Published: September 15, 2020.

\section{Introduction}

The growing demand for high-rate date services through wireless communication channels experienced in recent years motivates the design of multiple antenna wireless communication systems to transmit increase data rates without substantial bandwidth expansion. In particular, the antenna diversity can be used to improve the performance of wireless communication systems such as the code-division multiple-access (CDMA). First presented in [1] for two transmitting antennas and generalized in [2] and [3] for an arbitrary number of transmitting antennas, the orthogonal space-time block coding (STBC) is a remarkable technique which can provide full diversity gain with very low computational complexity.

However, a loss in capacity, characterized by the code rate and the number of receive antennas, is discussed shown in [4] and [5] for an arbitrary channel. Following the analysis in [3]-[5], a characterization based on the equivalent scalar additive white Gaussian noise (AWGN) channel multiplied by a coefficient, which is a function of the Frobenius norm of the channel matrix with multiple antennas, was given in [4] assuming a full code rate. The Shannon and outage capacity for the scalar AWGN channel were also given.

The Shannon capacity

$$
C=W \log _{2}(1+S N R),
$$

where $S N R$ is the signal-to-noise ratio and $W$ is the channel bandwidth, predicts the channel capacity $C$ for an AWGN channel with continuous-valued inputs and continuous-valued outputs. However, a channel employing the STBC with the pulse-amplitude modulation (PAM), phase shift keying (PSK) or quadrature-amplitude modulation (QAM) has discretevalued inputs and continuous-valued outputs, which imposes an additional constraint on the capacity calculation.

In this paper, we generalize the effective channel representation in [4] for all rate orthogonal STBCs, including the rate 0.5 STBCs $\mathbf{G}_{N}, N>2$ and the rate 0.75 STBCs $\mathbf{H}_{3}$ and $\mathbf{H}_{4}$, given in [2]. A new capacity calculation taking into account the constraint of discrete-valued inputs is presented here, as well as the capacity loss incurred by employing STBC.

In [6], an analysis of the bit error probability $(B E P)$ of $\mathbf{G}_{2}$ for $q$-ary PSK was presented for the Rayleigh fading channel using the probability density function (pdf) of the received signal phase from [7]. However, the BEP for $q$-PSK $(q>4)$ is very complex using this approach. In [8], a union bound on the symbol error probability (SEP) for the STBC was presented. A general form for the exact pair wise error 
probability of STBCs was obtained in [9] based on the moment generating function of the Gaussian tail function. In [10], the STBC was applied to a CDMA downlink, and a novel decoding algorithm was presented. In [11], the exact expression for the pair wise error probability in a flat Rayleigh fading channel was derived in terms of the symbol distance between two message vectors for quaternary phase-shift keying (QPSK),16-QAM, 64-QAM and 256-QAM. In [12], a unified approach to calculate the error rates of linearly modulated signals over generalized fading channels was presented. However, the results of both [11] and [12] are given in an open form that has to be evaluated via numerical integration.

In this paper, we analyze the error probability of STBCs for PAM, PSK, QAM modulated signals as a function of an SNR perspective based upon the equivalent scalar channel induced by the STBC. Using the pdf of the SNR, the closed form SEPS are given for various combinations of modulation and fading channels. Furthermore, these results are extended to a multiuser DS-CDMA system with STBC employing the generalized receiver. Expressions for the capacity and error probability of the DS-CDMA system with STBC are derived and analyzed.

\section{Channel Model}

\subsection{General channel model}

The channel model is the same as in [2]-[4]. Consider the wireless communication system with $N$ transmit and $M$ receive antennas. The channel is assumed to be quasistatic with flat fading, which means that the channel parameters are constant within one frame period, but are varied independently between frames. Furthermore, the perfect channel state information is assumed available at the receiver input, but the channel parameters are unknown at the transmitter.

Let $T$ represents the number of time slots used to transmit $S$ symbols. Hence, a general form for the transmission matrix of STBC is given as

$$
\mathbf{G}=\left(\begin{array}{cccc}
g_{11} & g_{21} & \cdots & g_{N 1} \\
g_{12} & g_{22} & \cdots & g_{N 2} \\
\cdots & \cdots & \cdots & \cdots \\
g_{1 T} & g_{2 T} & \cdots & g_{N T}
\end{array}\right),
$$

where $g_{i j}$ represent a linear combination of the signal constellation components and their conjugates, and are transmitted simultaneously by the $i$-th transmitting antenna in the $j$-th time slot for $i=1 \ldots N$ and $j$ $=1 \ldots T$. Since there are $S$ symbols transmitted over $T$ time slots, the code rate of the STBC is given by

$$
R=S / T \text {. }
$$

It is shown in [2], based on the theory of orthogonal designs, that full rate STBCs exists for any number of transmitting antennas using an arbitrary real constellation such as PAM. For an arbitrary complex constellation such as PSK, QAM, the half rate STBCs exist for any number of transmitting antennas, while full rate STBCs exist only for two transmitting antennas. As specific cases for two, three, and four transmitting antennas, the rate $1,0.5$, and 0.75 STBCs are given in [2], and are denoted as $\mathbf{G}_{2}, \mathbf{G}_{3}$, $\mathbf{G}_{4}, \mathbf{H}_{3}$, and $\mathbf{H}_{4}$, respectively. At a particular time $n T$, the received signal corresponding to the $n$-th input block spanning $T$ time slots takes the form

$$
\mathbf{Y}_{n T}=\mathbf{H G}^{T}+\mathbf{W}_{n T}
$$

where $\mathbf{Y}_{n T}$ is the $M \times T$ matrix; $\mathbf{H}$ is the $M \times N$ fading channel coefficient matrix with the independent identically distributed (i.i.d.) entries modelled as the circular complex Gaussian random variables; $\mathbf{G}^{T}$ is the transpose of $\mathbf{G}$ with the size $N \times T$, and $\mathbf{W}_{n T}$ is the $M$ $\times N$ receiver noise matrix with the i.i.d. entries modelled as the circular complex Gaussian random variables with zero mean and variance $\sigma_{W}=\Delta f \times \mathcal{N}_{0} / 2$ in each dimension, where $\Delta f$ is the bandwidth of linear system at the receiver input and $\mathscr{N}_{0} / 2$ is the AWGN power spectral density.

\subsection{Effective scaled AWGN channel}

In [4], an equivalent scaled AWGN channel induced by the STBC for the complex constellations was given as

$$
\mathbf{y}_{n T}=\|\mathbf{H}\|_{F}^{2} \mathbf{x}_{n T}+\mathbf{w}_{n T},
$$

where $\mathbf{y}_{n T}$ is the $S \times 1$ complex matrix after STBC decoding from the received matrix $\mathbf{Y}_{n T} ; \mathbf{x}_{n T}$ is the input $S \times 1$ complex input matrix with each entry having the energy $E_{S} / N, E_{s}$ is the maximum total transmited energy on the $N$ transmit antennas per symbol time; and $\mathbf{w}_{n T}$ is the complex Gaussian noise with the zero mean and variance $\|\mathbf{H}\|_{F}^{2} \mathcal{N}_{0} / 2$ in each real dimension;

$$
\|\mathbf{H}\|_{F}^{2}=\sum_{i=1}^{N} \sum_{j=1}^{M}\left\|h_{i j}\right\|^{2}
$$

is the squared Frobenius norm of $\mathbf{H}$; $h_{i j}$ is the channel gain from the $i$-th transmit antenna to the $j$-th receive antenna. Taking into account the code rate, the equivalent AWGN scaled channel with STBC is

$$
\mathbf{y}_{n T}=(1 / R)\|\mathbf{H}\|_{F}^{2} \mathbf{x}_{n T}+\mathbf{w}_{n T},
$$

where $\mathbf{w}_{n T}$ is the complex Gaussian noise with the zero mean and variance given as

$$
\operatorname{Var}=(1 / 2 R)\|\mathbf{H}\|_{F}^{2} \mathfrak{N}_{0}
$$


in each real dimension. Therefore, the effective instantaneous $S N R$ denoted as $\gamma_{s}$ at the receiver input takes the following form

$$
\gamma_{s}=\frac{E_{s}}{N R_{e} \mathcal{N}_{0}}\|\mathbf{H}\|_{F}^{2} .
$$

Let

$$
h=\frac{1}{R}\|\mathbf{H}\|_{F}^{2}=\sum_{i=1}^{N} \sum_{j=1}^{M} \frac{\left\|h_{i j}\right\|^{2}}{R},
$$

then the STBC channel model of (7) can be simplified to

$$
\mathbf{y}_{n T}=h \mathbf{x}_{n T}+\mathbf{w}_{n T}
$$

and $\gamma_{s}$ can be written as

$$
\gamma_{s}=\frac{E_{s}}{N_{\mathscr{N}} \mathcal{N}_{0}} h .
$$

\subsection{Distribution of the channel coefficients and $S N R$ for fading channels}

Rayleigh fading: With Rayleigh fading, the coefficients $h_{i j}$ can be modelled as the complex Gaussian variable with the zero mean and variance $\sigma_{w}^{2}$ in each dimension. The pdf of the coefficient $h$ is then the central chi-square distribution with $2 M N$ degrees of freedom

$$
f_{\text {Rayleigh }}(h)=\frac{R^{M N}}{\left(2 \sigma_{w}^{2}\right)^{M N} \Gamma(M N)} h^{M N-1} \exp \left\{-\frac{h R}{2 \sigma_{w}^{2}}\right\}, h \geq 0 .
$$

Consequently, the instantaneous SNR per symbol $\gamma_{s}$ is also chi-square distributed. Using a change of variables, the pdf of $\gamma_{s}$ is given by

$$
f_{\text {Rayleigh }}(h)=\frac{R^{M N}}{\bar{\gamma}_{c h}^{M N} \Gamma(M N)} \gamma_{s}^{M N-1} \exp \left\{-\frac{\gamma_{s}}{\bar{\gamma}_{c h}}\right\}, \gamma_{s} \geq 0
$$

where $\bar{\gamma}_{c h}$ is the average SNR per channel, which is assumed to be identical for all channels, i.e.

$$
\bar{\gamma}_{c h}=\frac{E_{s}}{N R_{e} \mathcal{N}_{0}} E\left[\left\|h_{i j}\right\|^{2}\right]=\frac{2 \sigma_{w}^{2} E_{s}}{N R_{e} \mathcal{N}_{0}},
$$

where $E[\cdot]$ is the mathematical expectation. It can easily be shown that the instantaneous $S N R$ per bit $\gamma_{b}$ has the same pdf except that

$$
\bar{\gamma}_{c h}=\frac{E_{s}}{N R_{e} N_{0} \log _{2} \mathcal{N}}
$$

for the $\mathcal{M}$-ary signal constellation.

Rician fading: For Rician fading, the coefficients $h_{i j}$ can be modelled as a complex Gaussian variable with the means $\mu_{I}$ and $\mu_{Q}$ for the real and imaginary parts, respectively, and the variance $\sigma_{w}^{2}$ in each dimension. In this case, $\|\mathbf{H}\|_{F}^{2}$ has a non-central chi-square distribution with $2 M N$ degrees of freedom. The pdf of $h$ is given by

$$
\begin{gathered}
f_{\text {Rician }}(h)=\frac{R}{2 \sigma_{w}^{2}}\left[\frac{R h}{s^{2}}\right]^{\frac{M N-1}{2}} \exp _{h \geq 0}\left\{-\frac{s^{2}+R h}{2 \sigma_{w}^{2}}\right\} I_{M N-1}\left(\frac{\sqrt{R h} s}{\sigma_{w}^{2}}\right), \\
h \geq
\end{gathered}
$$

where

$$
s^{2}=M N\left(\mu_{I}^{2}+\mu_{Q}^{2}\right)
$$

is the non-centrality parameter, $I_{\alpha}(x)$ is the $\alpha$-th order modified Bessel function of the first kind, which may be represented by the infinite series

$$
I_{m}(x)=\sum_{n=0}^{\infty} \frac{(0.5 x)^{n+2 k}}{k ! \Gamma(n+k+1)} .
$$

Introducing the Rician parameter

$$
\beta=\frac{\mu_{I}^{2}+\mu_{Q}^{2}}{2 \sigma_{w}^{2}},
$$

the pdf in (13) can be written in the following form

$$
\begin{gathered}
f_{\text {Rician }}(h)=\sum_{i=0}^{\infty} \frac{(M N \beta)^{i} \exp \{-M N \beta\} R^{M N+i}}{\Gamma(i+1) \Gamma(M N+i)\left(2 \sigma_{w}^{2}\right)^{M N+i}} \\
\times h^{M N+i-1} \exp \left\{-R h / 2 \sigma_{w}^{2}\right\}, \quad h \geq 0 .
\end{gathered}
$$

Using a change of variables, the pdf of the instantaneous $S N R$ takes the following form:

$$
\begin{gathered}
f_{\text {Rician }}(h)=\sum_{i=0}^{\infty} \frac{(M N \beta)^{i} \exp \{-M N \beta\} \gamma_{s}{ }^{M N+i-1} \exp \left\{-\gamma_{s} / \bar{\gamma}_{c}\right\}}{\Gamma(i+1) \Gamma(M N+i) \bar{\gamma}_{c}^{M N+i}}, \\
\gamma_{s} \geq 0,
\end{gathered}
$$

where $\bar{\gamma}_{c}$ is the average $S N R$ per channel, which is assumed to be identical for all channels, as given in (15).

Nakagami-m fading: For Nakagami-m fading with the integer $m,\left\|h_{i j}\right\|$ is the amplitude of the channel coefficient $h_{i j}$ and has a Nakagami- $m$ distribution with the variance $\sigma_{N}^{2}$ in each dimension. The random variable

$$
y=R^{-1}\left\|h_{i j}\right\|^{2}
$$

then has the pdf

$$
f(y)=\frac{R^{m}}{\left(2 \sigma^{2}\right)^{m} \Gamma(m)} y^{m-1} \exp \left\{-R y / 2 \sigma^{2}\right\},
$$

where $\sigma^{2}=\sigma_{N}^{2} / m$. Observing that the pdf for Nakagami- $m$ fading has the same form as the pdf for the Rayleigh fading but with $2 m$ degree in (13), a single Nakagami- $m$ fading channel is equivalent to an $m$ diversity system for the Rayleigh fading channel. It is then straightforward to show that the results for STBCs over the Nakagami- $m$ fading channels can be obtained by considering the Rayleigh fading channels with the channel diversity order increased from $M N$ to $m M N$. Consequently, the pdf of the instantneous $S N R$ per symbol $\gamma_{S}$ can be obtained direc- 
tly from (10) as

$$
f_{\text {Nakagami-m }}\left(\gamma_{s}\right)=\frac{1}{\bar{\gamma}_{c}^{m M N} \Gamma(m M N)} \gamma_{s}^{m M N-1} \exp \left\{-\gamma_{s} / \bar{\gamma}_{c}\right\},
$$

where $\bar{\gamma}_{c}$ is the average $S N R$ per channel, which is assumed to be identical for all channels, as in (15).

\section{Capacity Analysis of STBC}

\subsection{Shannon capacity over fading channels}

The capacity of a multiple antenna wireless communication system over a fading channel with continuous-valued inputs and continuous-valued outputs is given in [13] in the following form:

$$
C=E\left[\log _{2} \operatorname{det}\left(\mathbf{I}+\frac{E_{s}}{N_{e} \mathcal{N}_{0}} \mathbf{H H}^{T^{*}}\right)\right],[(b / s) / \mathrm{Hz}],
$$

where $E[\cdot]$ is the mathematical expectation; $\mathbf{I}$ is the identity matrix with $M$ dimension; $\operatorname{det}(\mathbf{x})$ denotes the determinant of the matrix $\mathbf{x}$, and the superscript $T^{*}$ denotes the matrix transpose and conjugate. The capacity of the equivalent STBC channel in (7) with the continuous-valued inputs and continuous-valued outputs for complex signals is given in [4] and [5] as

$$
\bar{C}=E\left[R \log _{2} \operatorname{det}\left(1+\frac{E_{s}}{R N_{e} \mathcal{N}_{0}}\|\mathbf{H}\|_{F}^{2}\right)\right]=E\left[R \log _{2}\left(1+\gamma_{s}\right)\right],
$$

Given the pdf of $\gamma_{s}$, the capacity of the equivalent STBC channel can be obtained based on

$$
\bar{C}=R \int_{0}^{\infty} \log _{2}\left(1+\gamma_{s}\right) f\left(\gamma_{s}\right) d \gamma_{s}, \quad[(b / s) / H z],
$$

\subsection{Capacity of $M$-ary signal constellations over fading channels}

Both (26) and (27) were obtained assuming continuous valued inputs. Here, we consider modulation channels with the discrete-valued multilevel phase inputs and continuous-valued outputs. Assuming maximum likelihood (ML) soft decoding with perfect channel state information at the receiver input, it is well known [14]-[16] that the capacity $C_{\text {STBC }}^{*}$ of the STBC channel (7) can be obtained by averaging the corresponding conditional capacity $\widetilde{C}^{*}(\mathbf{H})$ with respect to the joint pdf of the channel matrix $\mathbf{H}$. By doing so, the following expression for the capacity $C_{S T B C}^{*}$ of the fading channel is obtained

$C_{S T B C}^{*}=E\left[\widetilde{C}^{*}(\mathbf{H})\right]=\int \widetilde{C}^{*}(\mathbf{H}) f(\mathbf{H}) d \mathbf{H},[(b / s) / \mathrm{Hz}]$,

with

$$
\widetilde{C}^{*}(\mathbf{H})=R\left\{\log _{2} q-\frac{1}{q \pi R^{-1}\|\mathbf{H}\|_{F}^{2} \mathscr{N}_{0}^{\circ}}\right.
$$

$$
\begin{gathered}
\times \sum_{j=1}^{q} \int_{y \in C} \exp \left\{-\frac{\left\|y-R^{-1}\right\| \mathbf{H}\left\|_{F}^{2} \alpha_{j}\right\|^{2}}{R^{-1}\|\mathbf{H}\|_{F}^{2} \mathcal{N}_{0}}\right\} \\
\times \log _{2}\left[\sum _ { s = 1 } ^ { q } \operatorname { e x p } \left\{\frac{\left\|y-R^{-1}\right\| \mathbf{H}\left\|_{F}^{2} \alpha_{j}\right\|^{2}}{R^{-1}\|\mathbf{H}\|_{F}^{2} \mathfrak{N}_{0}^{\rho}}\right.\right. \\
\left.\left.\left.-\frac{\left\|y-R^{-1}\right\| \mathbf{H}\left\|_{F}^{2} \alpha_{s}\right\|^{2}}{R^{-1}\|\mathbf{H}\|_{F}^{2} \mathfrak{N}_{0}}\right\}\right] d y\right\},
\end{gathered}
$$

where $\alpha_{j}, j=1, \ldots, q$ is a real signal in the $q$-ary PAM constellation or complex in the $q$-ary PSK/QAM constellation, and $f(\mathbf{H})$ is the joint pdf of the $M \times N$ random elements of the channel matrix $\mathbf{H}$ for the fading channel.

Applying the channel model (11), (29) can be simplified to the one-dimensional integral containing the pdf of $h$

$$
C_{S T B C, R}^{*}=E\left[\widetilde{C}^{*}(h)\right]=\int \widetilde{C}^{*}(h) f(h) d h,[(b / s) / \mathrm{Hz}],
$$

where

$$
\begin{gathered}
\widetilde{C}^{*}(h)=R\left\{\log _{2} q-\frac{1}{q \pi h_{e} \mathcal{N}_{0}} \sum_{j=1}^{q} \int_{y \in C} \exp \left\{-\frac{\left\|\mathbf{y}-h \alpha_{j}\right\|^{2}}{h_{e} \mathcal{V}_{0}}\right\}\right. \\
\left.\quad \times \log _{2}\left[\sum_{s=1}^{q} \exp \left\{\frac{\left\|\mathbf{y}-h \alpha_{j}\right\|^{2}-\left\|\mathbf{y}-h \alpha_{s}\right\|^{2}}{h_{e} \mathcal{N}_{0}}\right\}\right] d \mathbf{y}\right\} .
\end{gathered}
$$

Note that (31) applies to both the real signal constellations such as PAM, and the complex signal constellations such as the PSK and QAM.

\subsection{Capacity comparison}

It is shown in [4] that the difference between (26) and (27) is the capacity loss incurred by us ing a STBC in the multiple-input multiple output (MIMO) wireless communication system over fading channel with the continued valued inputs. The capacity of MIMO fading channel with the PAM, PSK, QAM is given in [15] as

$$
C_{M, N}^{*}=\int \widetilde{C}_{M, N}^{*}(\mathbf{H}) f(\mathbf{H}) d \mathbf{H}, \quad[(b / s) / H z],
$$

where

$$
\begin{gathered}
\widetilde{C}_{M, N}^{*}(\mathbf{H})=N \log _{2} q-q^{-N}\left(\pi \mathcal{N}_{0}\right)^{-M} \\
\times \sum_{\mathbf{x} \in(A x)^{N}} \int_{\mathbf{y} \in C} \exp \left\{-\frac{\|\mathbf{y}-\mathbf{H x}\|^{2}}{\mathcal{N}_{0}}\right\} \\
\times \log _{2}\left[\sum_{\mathbf{x}^{\prime} \in(A x)^{N}} \exp \left\{-\frac{\|\mathbf{y}-\mathbf{H} \mathbf{x}\|^{2}-\mid \mathbf{y}-\mathbf{H} \mathbf{x}^{\prime} \|^{2}}{\mathscr{N}_{0}}\right\} d \mathbf{y} .\right.
\end{gathered}
$$

Here, $N$ and $M$ are the number of transmit and receive antennas, respectively;

$$
A x \equiv\left\{\alpha_{1}, \ldots, \alpha_{q}\right\}
$$

is the $q$-ary complex signal constellation; $(A x)^{N}$ is the $\mathrm{N}$-fold Cartesian product of $\mathrm{Ax}$ with itself; the coded vector 


$$
\mathbf{x} \equiv\left[x_{1}, \ldots, x_{N}\right] \in(A x)^{N}
$$

is the $q^{N}$ - variate random variable with outcomes taking values from the expanded signal constellation $(A x)^{N} ;$ and

$$
\mathbf{y} \equiv\left[y_{1}, \ldots, y_{N}\right]^{T}
$$

is the $M$-dimensional output vector of the receive antennas. It can easily be shown that the second terms in (32) and (34) vanish as the SNR increases. This fact implies the capacity of a MIMO fading channel approaches $N \log _{2} q$ bit/channel use while the capacity with STBC approaches only $R \log _{2} q$ bit /channel use for the large SNR. While the capacity loss of $(N-R) \times \log _{2} q$ bit/channel incurred by using STBC is fairly significant, it will be shown below that the SNR threshold for reliable data transmission is reduced because of the STBC diversity gain.

\section{Analysis of Error Probability over Fading Channels}

\subsection{Error probability with Rayleigh fading}

Let $P_{q}^{\text {error }}\left(\gamma_{s}\right)$ is the error probability of $q$-ary signal constellation with STBC in AWGN channel. The error probability with the Rayleigh fading can be obtained by averaging $P_{q}^{\text {error }}\left(\gamma_{s}\right)$ over the pdf of $\gamma_{s}$

$$
P_{S T B C, q}^{\text {error }}\left(\gamma_{s}\right)=\int_{0}^{\infty} P_{q}^{\text {error }}\left(\gamma_{s}\right) f_{\text {Rayleigh }}\left(\gamma_{s}\right) d \gamma_{s} .
$$

Note, $P_{q}^{\text {error }}\left(\gamma_{s}\right)$ can be SEP or BEP, respectively.

Error probability for PAM: Since full-rate STBCs exist for any number of transmitting antennas using the real PAM constellation [2], $R=1$ for $q$-ary PAM. The average SEP for PAM over AWGN channel is [7]

$$
P_{q}^{\text {error }}\left(\gamma_{s}\right)=2\left(1-q^{-1}\right) Q\left[\sqrt{6\left(q^{2}-1\right)^{-1} \gamma_{s}}\right],
$$

where $Q(\cdot)$ is the Gaussian tail function. Substituting (14) and (39) into (38), the average SEP for PAM is

$$
\begin{aligned}
P_{S T B C, \text { PAM }, q}^{\text {error }}\left(\gamma_{s}\right)=\int_{0}^{\infty} 2\left(1-q^{-1}\right) Q\left[\sqrt{6\left(q^{2}-1\right)^{-1} \gamma_{s}}\right] \\
\times \frac{\gamma_{s}^{M N-1}}{\bar{\gamma}_{c}^{M N} \Gamma(M N)} \exp \left\{-\gamma_{s} / \bar{\gamma}_{c}\right\} d \gamma_{s} .
\end{aligned}
$$

To evaluate the integral in (40), the following integral function can be employed:

$$
\begin{gathered}
g(L)=\int_{0}^{\infty} Q(\sqrt{a x}) x^{L-1} \exp \{-x / u\} d x \\
=0.5 u^{L} \Gamma(L)\left[1-\sum_{k=0}^{L-1} \mu\left(\frac{1-\mu^{2}}{4}\right)^{k}\left(\begin{array}{c}
2 k \\
k
\end{array}\right)\right],
\end{gathered}
$$

where

$$
\mu=\sqrt{\frac{a u}{2+a u}} .
$$

The proof is given in Appendix 1. The closed form of the SEP for PAM is then given by

$$
P_{S T B C, P A M, q}^{\text {error }}\left(\gamma_{s}\right)=\left(1-q^{-1}\right)\left[1-\sum_{k=0}^{M N-1} \mu\left(\frac{1-\mu^{2}}{4}\right)^{k}\left(\begin{array}{c}
2 k \\
k
\end{array}\right)\right] \text {, }
$$

where

$$
\mu=\sqrt{\frac{3 \bar{\gamma}_{c}}{q^{2}-1+3 \bar{\gamma}_{c}}} .
$$

Error probability for PSK: Based on the equivalent scalar AWGN channel model presented before, the error probability for $q$-ary PSK is equivalent to the analysis in [7] and [17] for adaptive reception of multiphase signals in Rayleigh fading but with $M N$ branch diversity. This approach was also employed in [6]. Following the same steps as in [17], the SEP is given by

$$
\begin{aligned}
P_{S T B C, q}^{\text {error }}= & \frac{(-1)^{M N-1}\left(1-\mu^{2}\right)^{M N}}{\pi \Gamma(M N)}\left\{\frac { \partial ^ { M N - 1 } } { \partial s ^ { M N - 1 } } \left[\frac { 1 } { s - \mu ^ { 2 } } \left(\frac{\pi}{M}\right.\right.\right. \\
& \times(M-1)-\frac{\mu \sin (\pi / M)}{\sqrt{s-\mu^{2} \cos ^{2}(\pi / M)}} \\
\times & \left.\left.\left.\cot ^{-1}\left(-\frac{\mu \cos (\pi / M)}{\sqrt{s-\mu^{2} \cos ^{2}(\pi / M)}}\right)\right)\right]\right\}\left.\right|_{s=1},
\end{aligned}
$$

where

$$
\mu=\sqrt{\frac{\bar{\gamma}_{c}}{1+\bar{\gamma}_{c}}}
$$

and the notation

$$
\left.\frac{\partial^{M N-1}}{\partial s^{M N-1}} f(s, \mu)\right|_{s=1}
$$

denotes the $(M N-1)$-th partial derivative of the function $f(s, \mu)$ evaluated at $s=1$. Note that the coherent detection with perfect channel state information at the receiver input is assumed in (45). Following approach in [7], [17], performing the differentiation indicated in (45) and evaluating the resulting function at $s=1$ for $q=2$ and 4 , we obtain the following closed form BEPs for the binary phase shift keying (BPSK) and QPSK

$$
\begin{gathered}
P_{S T B C, 2}^{\text {error }}=0.5\left[1-\mu \sum_{k=0}^{M N-1}\left(\begin{array}{c}
2 k \\
k
\end{array}\right)\left(\frac{1-\mu^{2}}{4}\right)^{k}\right], \\
P_{S T B C, 4}^{\text {error }}=0.5\left[1-\frac{\mu}{\sqrt{2-\mu^{2}}} \sum_{k=0}^{M N-1}\left(\begin{array}{c}
2 k \\
k
\end{array}\right)\left(\frac{1+\mu^{2}}{4-2 \mu^{2}}\right)^{k}\right],
\end{gathered}
$$


respectively. Note that the Gray coding was assumed in the BEP calculation for QPSK. The same procedure can be applied to calculate the $S E P$ for $q$-ary PSK at $q=8,16,32$, however, the expressions are not as simple as in (47) and (48). In the remainder of this section we employ (38) in order to derive a simpler expression for the error probability.

It is well known [7] that the BEP for BPSK and QPSK over the additive white Gaussian noise channel are given as

$$
\begin{aligned}
& P_{B P S K}^{\text {error }}\left(\gamma_{s}\right)=Q\left(\sqrt{2 \gamma_{s}}\right), \\
& P_{Q P S K}^{\text {error }}\left(\gamma_{s}\right)=Q\left(\sqrt{\gamma_{s}}\right),
\end{aligned}
$$

respectively. As shown in [18], the exact SEP of $M$ ary PSK for the AWGN channel can be presented in the following form

$$
\begin{gathered}
P_{P S K, A W G N, M}^{\text {error }}\left(\gamma_{s}\right) \\
=2 Q\left[\sqrt{2 \gamma_{s}} \sin (\pi / q)\right]-\frac{1}{\pi} \int_{\pi / 2-\pi / q}^{\pi / 2} \exp \left\{-\gamma_{s} \frac{\sin ^{2}(\pi / q)}{\cos ^{2} \theta} d \theta\right\}
\end{gathered}
$$

For large $S N R$ and large values of $q$,the SEP of $q$-ary PSK in the AWGN channel can be approximated as

$$
P_{P S K, A W G N, q}^{\text {error }}\left(\gamma_{s}\right) \approx 2 Q\left[\sqrt{2 \gamma_{s}} \sin (\pi / q)\right]
$$

and the equivalent $B E P$ is given by

$$
P_{P S K, A W G N, q}^{\text {error }}\left(\gamma_{s}\right) \approx \frac{S E P_{q}}{\log q},
$$

where the Gray coding is assumed. This approximation is good for large values of $q$, however, for $q=2$ there is a difference in the factor of 2 with the exact probability given in (47).

By substituting for $P_{P S K, A W G N, q}^{\text {error }}\left(\gamma_{s}\right)$ in (52) and using (41), (38) can be written in the following form

$$
\begin{gathered}
P_{S T B C, P S K, q}^{\text {error }}\left(\gamma_{s}\right) \\
\approx \int_{0}^{\infty} 2 Q\left[\sqrt{2 \gamma_{s}} \sin (\pi / q)\right] \frac{\gamma_{s}^{M N-1}}{\bar{\gamma}_{c}^{M N} \Gamma(M N)} \exp \left\{-\gamma_{s} / \bar{\gamma}_{c}\right\} d \gamma_{s} \\
=1-\sum_{k=0}^{M N-1} \mu\left(\frac{1-\mu^{2}}{4}\right)^{k}\left(\begin{array}{c}
2 k \\
k
\end{array}\right),
\end{gathered}
$$

where

$$
\mu=\sqrt{\frac{\sin ^{2}\left(\pi \gamma_{c} / q\right)}{1+\sin ^{2}\left(\pi \gamma_{c} / q\right)}} .
$$

Therefore, the BEP can be approximated as

$$
P_{S T B C, P S K, q}^{\text {error }}\left(\gamma_{s}\right) \approx \frac{1}{\log q}\left[1-\sum_{k=0}^{M N-1} \mu\left(\frac{1-\mu^{2}}{4}\right)^{k}\left(\begin{array}{c}
2 k \\
k
\end{array}\right)\right] \text {. }
$$

By substituting for $P_{B P S K}^{\text {error }}\left(\gamma_{s}\right)$ or $P_{Q P S K}^{\text {error }}\left(\gamma_{s}\right)$ in (49) and (50) and using (41), the exact BEP for BPSK and QPSK can be derived from (38) as

$$
P_{S T B C, P S K, q}^{\text {error }}\left(\gamma_{s}\right)=\frac{1}{2}\left[1-\sum_{k=0}^{M N-1} \mu\left(\frac{1-\mu^{2}}{4}\right)^{k}\left(\begin{array}{c}
2 k \\
k
\end{array}\right)\right],
$$

where

$$
\mu=\sqrt{\frac{\bar{\gamma}_{c}}{1+\bar{\gamma}_{c}}}
$$

for BPSK and

$$
\mu=\sqrt{\frac{\bar{\gamma}_{c}}{2+\bar{\gamma}_{c}}}
$$

for QPSK, respectively. It can easily be shown that (57) is equivalent to (47) and (48). Using (56), approximations for the BEPs of BPSK and QPSK can be obtained as

$$
P_{S T B C, B P S K, 2}^{\text {error }} \approx 1-\sum_{k=0}^{M N-1} \mu\left(\frac{1-\mu^{2}}{4}\right)^{k}\left(\begin{array}{c}
2 k \\
k
\end{array}\right)
$$

with $\mu$ given by (58) and

$$
P_{S T B C, Q P S K, 4}^{\text {error }} \approx \frac{1}{2}\left[1-\sum_{k=0}^{M N-1} \mu\left(\frac{1-\mu^{2}}{4}\right)^{k}\left(\begin{array}{c}
2 k \\
k
\end{array}\right)\right]
$$

with $\mu$ given by (59), respectively. As expected, this approximation is unsuitable for BPSK, but equation (61) brings us the exact BEP for QPSK. It is shown later that this approximation is very accurate for $q>4$ and large SNR.

Error probability for QAM: Rectangular QAM signal constellations are frequently employed because they are equivalent to two PAM signals on qua drature carriers. For $q$-ary, $q=2^{k}$ ( $k$ is even), rectangular QAM, the SEP is given in [7] as

$$
P_{q}^{\text {error }}=1-\left[1-P_{\sqrt{q}}^{\text {error }}\left(\gamma_{s}\right)\right]^{2},
$$

where

$$
P_{\sqrt{q}}^{e r r o r}\left(\gamma_{s}\right)=2\left(1-q^{-0.5}\right) Q\left(\sqrt{\frac{3}{q-1} \gamma_{s}}\right) .
$$

By substituting for $P_{q}^{\text {error }}$ in (63), (38) can be written as

$$
\begin{gathered}
P_{\text {STBC,PAM }, \sqrt{q}}^{\text {error }}\left(\gamma_{s}\right)=\int_{0}^{\infty} 2\left(1-q^{-0.5}\right) Q\left(\sqrt{\frac{3}{q-1} \gamma_{s}}\right) \\
\times \frac{\gamma_{s}^{M N-1} \exp \left\{\gamma_{s} / \bar{\gamma}_{c}\right\}}{\bar{\gamma}_{c}^{M N} \Gamma(M N)} d \gamma_{s} .
\end{gathered}
$$

Using (41), the closed-form SEP for the rectangular $q$-ary QAM is then given by

$$
P_{S T B C, Q A M, q}^{\text {error }}\left(\gamma_{s}\right)=1-\left[1-P_{S T B C, P A M, \sqrt{q}}^{\text {error }}\left(\gamma_{s}\right)\right]^{2},
$$

where

$$
P_{S T B C, P A M, \sqrt{q}}^{\text {error }}\left(\gamma_{s}\right)=\left(1-q^{-0.5}\right)\left[1-\sum_{k=0}^{M N-1} \mu\left(\frac{1-\mu^{2}}{4}\right)^{k}\left(\begin{array}{c}
2 k \\
k
\end{array}\right)\right]
$$


and

$$
\mu=\sqrt{\frac{3 \bar{\gamma}_{c}}{2 q-2+3 \bar{\gamma}_{c}}} .
$$

\subsection{Error probability over Rician fading}

Substituting $f_{\text {Rician }}\left(\gamma_{s}\right)$ given by (22) in (38) instead of $f_{\text {Rayleigh }}\left(\gamma_{s}\right)$ and following the same procedure used previously, the SEP over Rician fading channel can be presented in the following form

$$
\begin{aligned}
P_{S T B C, \text { Rician }, q}^{\text {error }}\left(\gamma_{s}\right)=\sum_{n=0}^{\infty} \frac{(M N \beta)^{n} \exp \{-M N \beta\} \lambda}{\Gamma(n+1)} \\
\times\left[1-\sum_{k=0}^{M N+n-1} \mu\left(\frac{1-\mu^{2}}{4}\right)^{k}\left(\begin{array}{c}
2 k \\
k
\end{array}\right)\right]
\end{aligned}
$$

where

$$
\mu=\sqrt{\frac{3 \bar{\gamma}_{c}}{q^{2}-1+3 \bar{\gamma}_{c}}} \text { and } \lambda=1-q^{-1}
$$

for q-ary PAM,

$$
\mu=\sqrt{\frac{\sin ^{2}\left(\pi \gamma_{c} / q\right)}{1+\sin ^{2}\left(\pi \gamma_{c} / q\right)}}
$$

with $\lambda=0.5$ at $q=2$ and $\lambda=1$ at $q>2$ for $q$-ary PSK, and

$$
\mu=\sqrt{\frac{3 \bar{\gamma}_{c}}{2 q-2+3 \bar{\gamma}_{c}}} \text { and } \lambda=1-q^{-0.5}
$$

for $\sqrt{q}$-ary PAM in $q$-ary rectangular QAM. The SEP of $q$-ary rectangular QAM can then be calculated using (65). Note that each term in the second part of (68) is a monotonically decreasing function of $i$, and is strictly smaller than 1 for all $i$. The truncation of the first $L$ terms will introduce an error of at most

$$
\text { Error }=\frac{\lambda \mu\left(1-\mu^{2^{M N+L}}\right.}{1-\sum_{k=0}^{L} \frac{(M N K)^{k} \exp \{-M N K\}}{\Gamma(k+1)}}
$$

in the probability of error. The proof is given in Appendix II.

\subsection{Error probability under Nakagami-m fading}

The probability of error over Nakagami- $m, m$ is the integer, can be obtained from the results presented in Section 4.1 by increasing the diversity order from $M N$ to $m M N$. The SEP for STBC over a Nakagami$m$ fading channel is then

$$
P_{S T B C, \text { Nakagami } q}^{\text {error }}\left(\gamma_{S}\right)=\lambda\left[1-\sum_{k=0}^{M N+n-1} \mu\left(\frac{1-\mu^{2}}{4}\right)^{k}\left(\begin{array}{c}
2 k \\
k
\end{array}\right)\right],
$$

where $\mu$ and $\lambda$ for $q$-ary PAM, $q$-ary PAM, and $\sqrt{q}$ - ary PAM in $q$-ary rectangular QAM are given by (69)-(71), respectively. The SEP of $q$-ary rectangular QAM can be calculated by (65).

\section{Extension to STBC DS-CDMA}

There is a great interest in the application of STBCs to practical wireless communications systems constructed based on the generalized approach to signal processing in noise and employing the generalized receiver.

\subsection{Generalized receiver}

The generalized receiver is constructed in accordance with the generalized approach to signal processing in noise [19]-[21]. The generalized approach to signal processing in noise introduces an additional noise source that does not carry any information about the parameters of desired transmitted signal with the purpose to improve the signal processing system performance. This additional noise can be considered as the reference noise without any information about the parameters of the signal to be detected.

The jointly sufficient statistics of the mean and variance of the likelihood function is obtained under the generalized approach to signal processing in noise employment, while the classical and modern signal processing theories can deliver only a sufficient statistics of the mean or variance of the likelihood function. Thus, the generalized approach to signal processing in noise implementation allows us to obtain more information about the parameters of the desired transmitted signal incoming at the generalized receiver input. Owing to this fact, the detectors constructed based on the generalized approach to signal processing in noise technology are able to improve the signal detection performance of signal processing systems in comparison with employment of other conventional detectors.

The generalized receiver (GR) consists of three channels (see Fig.1): the GR correlation detector channel (GR CD) - the preliminary filter (PF), the multipliers 1 and 2, the model signal generator (MSG); the GR energy detector channel (GR ED) the $\mathrm{PF}$, the additional filter (AF), the multipliers 3 and 4 , the summator 1 ; and the GR compensation channel (GR CC) - the summators 2 and 3, the accumulator 1. The threshold apparatus (THRA) device defines the GR threshold. As we can see from Fig.1, there are two band-pass filters, i.e. the linear systems, at the GR input, namely, the PF and AF. We assume for simplicity that these two filters or linear systems have the same amplitude-frequency characteristics or impulse responses. The AF central frequency is detuned relative to the PF central frequency.

There is a need to note the PF bandwidth is mat- 
ched with the transmitted signal bandwidth. If the detuning value between the PF and AF central frequencies is more than 4 or 5 times the transmitted signal bandwidth to be detected, i.e. $4 \div 5 \Delta f_{s}$, where $\Delta f_{s}$ is the transmitted signal bandwidth, we can believe that the processes at the PF and AF outputs are uncorrelated because the coefficient of correlation between them is negligible (not more than 0.05). This fact was confirmed experimentally in [22] and [23] independently. Thus, the transmitted signal plus noise can be appeared at the GR PF output and the noise only is appeared at the GR AF output. The stochastic processes at the GR AF and GR PF outputs present the input stochastic samples from two independent frequency-time regions. If the discrete-time noise $w_{i}[k]$ at the GR PF and GR AF inputs is Gaussian, the discrete-time noise $\zeta_{i}[k]$ at the GR PF output is Gaussian too, and the reference discrete-time noise $\eta_{i}[k]$ at the GR AF output is Gaussian owing to the fact that the GR PF and GR AF are the linear systems and we believe that these linear systems do not change the statistical parameters of the input process. Thus, the GR AF can be considered as a generator of the reference noise with a priori information a "no" transmitted signal (the reference noise sample) [21, Chapter 5]. The noise at the GR PF and GR AF outputs can be presented as

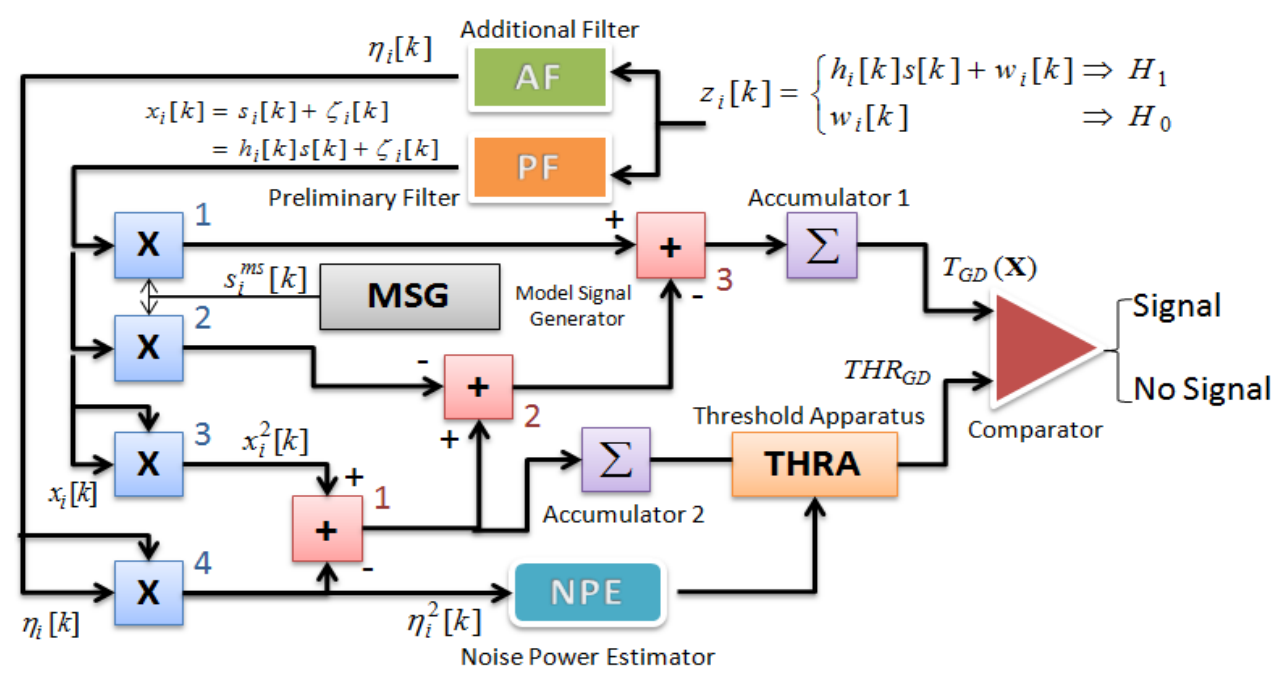

Fig. 1. Generalized receiver structure.

$$
\left\{\begin{array}{l}
\zeta_{i}[k]=\sum_{m=-\infty}^{\infty} g_{P F}[m] w_{i}[k-m] ; \\
\eta_{i}[k]=\sum_{m=-\infty}^{\infty} g_{A F}[m] w_{i}[k-m],
\end{array}\right.
$$

where $g_{P F}[m]$ and $g_{A F}[m]$ are the impulse responses of the GR PF and GR AF, respectively.

In a general, under practical implementation of any detector in wireless communication system with sensor array, the bandwidth of the spectrum to be sensed is defined. Thus, the GR AF bandwidth and central frequency can be assigned, too (this bandwidth cannot be used by the transmitted signal because it is out of its spectrum). The case when there are interfering signals within the GR AF bandwidth, the action of this interference on the GR detection performance, and the case of non-ideal condition when the noise at the GR PF and GR AF outputs is not the same by statistical parameters are discussed in [24] and [25].

Under the hypothesis $\mathscr{H}_{1}$ ("a yes" transmitted signal), the GR CD generates the signal component $s_{i}^{m}[k] s_{i}[k]$ caused by interaction between the model signal $s_{i}^{m}[k]$, forming at the MSG output, and the incoming signal $s_{i}[k]$, and the noise component $s_{i}^{m}[k]$ $\times \zeta_{i}[k]$ caused by interaction between the model signal $s_{i}^{m}[k]$ and the noise $\zeta_{i}[k]$ at the PF output. GR ED generates the transmitted signal energy $s_{i}^{2}[k]$ and the random component $s_{i}[k] \zeta_{i}[k]$ caused by interaction between the transmitted signal $s_{i}[k]$ and the noise $\zeta_{i}[k]$ at the PF output. The main purpose of the GR $\mathrm{CC}$ is to cancel completely in the statistical sense the GR CD noise component $s_{i}^{m}[k] \zeta_{i}[k]$ and the GR ED random component $s_{i}[k] \zeta_{i}[k]$ based on the same 
nature of the noise $\zeta_{i}[k]$. The relation between the transmitted signal to be detected $s_{i}[k]$ and the model signal $s_{i}^{m}[k]$ is defined as:

$$
s_{i}^{m}[k]=\mu s_{i}[k],
$$

where $\mu$ is the coefficient of proportionality.

The main functioning condition under the GR employment in any signal processing system including the communication one with radar sensors is the equality between the parameters of the model signal $s_{i}^{m}[k]$ and the incoming signal $s_{i}[k]$, for example, by amplitude. Under this condition it is possible to cancel completely in the statistical sense the noise component $s_{i}^{m}[k] \zeta_{i}[k]$ of the GR CD and the random component $s_{i}[k] \zeta_{i}[k]$ of the GR ED. Satisfying the GR main functioning condition given by (75), $s_{i}^{m}[k]=$ $s_{i}[k], \mu=1$, we are able to detect the transmitted signal with the high probability of detection at the low SNR and define the transmitted signal parameters with high accuracy.

Practical realization of this condition (75) at $\mu \rightarrow$ 1 requires increasing in the complexity of GR structure and, consequently, leads us to increasing in computation cost. For example, there is a need to employ the amplitude tracking system or to use the offline data samples processing. Under the hypothesis $\mathscr{H}_{0}$ ("a no" transmitted signal), satisfying the main GR functioning condition (75) at $\mu \rightarrow 1$ we obtain only the background noise $\eta_{i}^{2}[k]-\zeta_{i}^{2}[k]$ at the GR output.

Under practical implementation, the real structure of GR depends on specificity of signal processing systems and their applications, for example, the radar sensor systems, adaptive wireless communication systems, cognitive radio systems, satellite communication systems, mobile communication systems and so on. In the present paper, the GR circuitry (Fig.1) is demonstrated with the purpose to explain the main functioning principles. Because of this, the GR flowchart presented in the paper should be considered under this viewpoint. Satisfying the GR main functioning condition (75) at $\mu \rightarrow 1$, the ideal case, for the wireless communication systems with radar sensor applications we are able to detect the transmitted signal with very high probability of detection and define accurately its parameters.

In the present paper, we discuss the GR implementation in communication systems using the radar sensor array. Since the presented GR test statistics is defined by the signal energy and noise power, the equality between the parameters of the model signal $s_{i}^{m}[k]$ and transmitted signal to be detected $s_{i}[k]$, in particular by amplitude, is required that leads us to high circuitry complexity in practice. For example, there is a need to employ the amplitude tracking system or off-line data sample processing. Detailed discussion about the main GR functioning principles if there is no a priori information and there is an uncertainty about the parameters of transmitted signal, $i$. e., the transmitted signal parameters are random, can be found in [19] and [20, Chapter 6, pp.611-621 and Chapter 7, pp. 631-695].

The complete matching between the model signal $s_{i}^{m}[k]$ and the incoming signal $s_{i}[k]$, for example by amplitude, is a very hard problem in practice because the incoming signal $s_{i}[k]$ depends on both the fading and the transmitted signal parameters and it is impractical to estimate the fading gain at the low $S N R$. This matching is possible in the ideal case only. The GD detection performance will be deteriorated under mismatching in parameters between the model signal $s_{i}^{m}[k]$ and the transmitted signal $s_{i}[k]$ and the impact of this problem is discussed in [26][29], where a complete analysis about the violation of the main GR functioning requirements is presented. The GR decision statistics requires an estimation of the noise variance $\sigma_{\eta}^{2}$ using the reference noise $\eta_{i}[k]$ at the AF output.

Under the hypothesis $\mathscr{H}_{1}$, the signal at the GR PF output, see Fig. 1, can be defined as

$$
x_{i}[k]=s_{i}[k]+\zeta_{i}[k] \text {, }
$$

where $\zeta_{i}[k]$ is the noise at the PF output and

$$
s_{i}[k]=h_{i}[k] s[k],
$$

where $h_{i}[k]$ are the channel coefficients. Under the hypothesis $\mathscr{H}_{0}$ and for all $i$ and $k$, the process $x_{i}[k]=$ $\zeta_{i}[k]$ at the PF output is subjected to the complex Gaussian distribution and can be considered as the i.i.d. process.

In the ideal case, we can think that the signal at the GR AF output is the reference noise $\eta_{i}[k]$ with the same statistical parameters as the noise $\zeta_{i}[k]$. In practice, there is a difference between the statistical parameters of the noise $\eta_{i}[k]$ and $\zeta_{i}[k]$. How this difference impacts on the GR detection performance is discussed in detail in [20, Chapter 7, pp. 631-695] and in [26]-[29],

The decision statistics at the GR output presented in [19] and [20, Chapter 3] is extended for the case of antenna array when an adoption of multiple antennas and antenna arrays is effective to mitigate the negative attenuation and fading effects. The GR decision statistics can be presented in the following 
form:

$$
\begin{gathered}
T_{G R}(\mathbf{X})=\sum_{k=0}^{N-1} \sum_{i=1}^{M} 2 x_{i}[k] s_{i}^{m}[k] \\
-\sum_{k=0}^{N-1} \sum_{i=1}^{M} x_{i}^{2}[k]+\sum_{k=0}^{N-1} \sum_{i=1}^{M} \eta_{i}^{2}[k] \underset{\mathscr{H}_{0}}{\underset{\mathcal{H}_{1}}{>}} T H R_{G R},
\end{gathered}
$$

where

$$
\mathbf{X}=[\mathbf{x}(0), \ldots, \mathbf{x}(N-1)]
$$

is the vector of the random process at the GR PF output and $T H R_{G R}$ is the GR detection threshold.

Under the hypotheses $\mathscr{H}_{1}$ and $\mathscr{H}_{0}$ and when the amplitude of the transmitted signal is equal to the amplitude of the model signal, $s_{i}^{m}[k]=s_{i}[k], \mu=1$, the GR decision statistics $T_{G D}(\mathbf{X})$ takes the following form in the statistical sense, respectively:

$$
\left\{\begin{array}{l}
\mathscr{H}_{1}: T_{G D}(\mathbf{X})=\sum_{k=0}^{N-1} \sum_{i=1}^{M} s_{i}^{2}[k]+\sum_{k=0}^{N-1} \sum_{i=1}^{M} \eta_{i}^{2}[k]-\sum_{k=0}^{N-1} \sum_{i=1}^{M} \zeta_{i}^{2}[k], \\
\mathscr{H}_{0}: T_{G D}(\mathbf{X})=\sum_{k=0}^{N-1} \sum_{i=1}^{M} \eta_{i}^{2}[k]-\sum_{k=0}^{N-1} \sum_{i=1}^{M} \zeta_{i}^{2}[k] .
\end{array}\right.
$$

In (80) the term $\sum_{k=0}^{N-1} \sum_{i=1}^{M} s_{i}^{2}[k]=E_{s}$ corresponds to the average transmitted signal energy, and the term $\sum_{k=0}^{N-1} \sum_{i=1}^{M} \eta_{i}^{2}[k]-\sum_{k=0}^{N-1} \sum_{i=1}^{M} \zeta_{i}^{2}[k]$ is the background noise at the GR output. The GR output background noise is a difference between the noise power at the GR PF and GR AF outputs. Practical implementation of the GR decision statistics requires an estimation of the noise variance $\sigma_{\eta}^{2}$ using the reference noise $\eta_{i}[k]$ at the AF output.

\subsection{System model}

To facilitate the analysis, we generalize the CDMA multiple access interference model from [30] and [31] to accommodate multiple antennas. The system model is presented in Fig.2. In the transmitter, $S$ information symbols for $K$ users are encoded by the respective STBC encoders, and then spread by each user's pseudonoise code, modulated and transmitted from $N$ transmitting antennas over the symbol duration $T$, simultaneously. At the receivers, each user has $M$ receive antennas, and the filtered signals are first dispread, and then sent to STBC decoders. The symbol decisions are made based on the $M$ STBC decoder outputs. The signal at the $j$-th receive input antenna is given by

$$
z_{j}(t)=\sum_{n=1}^{N} \sum_{k=1}^{K} \sqrt{2 P_{k}} h_{k n j}(t) a_{k}\left(t-\tau_{k n j}\right) b_{n k}\left(t-\tau_{k n j}\right)
$$

$$
\times \cos \left[\omega_{c}\left(t-\tau_{k n j}\right)\right]+w(t),
$$

where $h_{k n j}(t)$ is the channel coefficient from the $n$-th transmitting antenna to the $j$-th receive antenna for the $k$-th user; $P_{k}=E_{s} / T_{s}$ is the symbol power of the $k$-th user; $E_{s}$ is the symbol energy; $T_{s}$ is the symbol duration; $a_{k}(t)$ is the $\mathrm{PN}$ spreading chip sequence with chip duration $T_{c} ; w(t)$ is the zero mean AWGN with the power spectral density $\mathcal{N}_{0} / 2$ in each real dimension; $\tau_{k n j}$ is the time delay from the $n$-th transmitting antenna to the $j$-th receive antenna; $\omega_{c}$ is the carrier frequency; $b_{n k}(t)$ is the encoded signal transmitted from the $n$-th antenna of user $k$. Binary modulation is assumed in this model.

\subsection{Channel model analysis}

To facilitate the analysis, we assume the first user is the desired one, without loss of generality. The dispread signal input to the $j$-th STBC decoder for the $i$-th received symbol is

$$
\begin{gathered}
\hat{z}_{1 j}(t)=\sum_{q=1}^{N} \int_{(i-1) T_{s}}^{i T_{s}}\left[\sqrt{2 P_{k}} \sum_{n=1}^{N} \sum_{k=1}^{K} h_{k n j}(t) a_{k}\left(t-\tau_{k n j}\right)\right. \\
\left.\times b_{n k}\left(t-\tau_{k n j}\right) \cos \left[\omega_{c}\left(t-\tau_{k n j}\right)\right]+w(t)\right] a_{1}\left(t-\tau_{1 q j}\right) \\
\times \cos \left[\omega_{c}\left(t-\tau_{1 q j}\right)\right] d t \\
=\sqrt{\frac{P_{1}}{2}} T_{s} \sum_{n=1}^{N} h_{1 n j}^{(i)} b_{n 1}^{(i)}+\int_{(i-1) T_{s}}^{i T_{s}}\left[\sqrt{2 P_{k}} \sum_{n=1}^{N} \sum_{\substack{q=1 \\
q \neq n}}^{N} h_{1 n j}(t) a_{1}\left(t-\tau_{1 n j}\right)\right. \\
\left.\times b_{n 1}\left(t-\tau_{1 n j}\right) \cos \left[\omega_{c}\left(t-\tau_{1 n j}\right)\right]\right] a_{1}\left(t-\tau_{1 n j}\right) \cos \left[\omega_{c}(t\right. \\
\left.\left.-\tau_{1 n j}\right)\right] d t+\sum_{q=1}^{N} \int_{(i-1) T_{s}}^{i T_{s}} w(t) a_{1}\left(t-\tau_{1 n j}\right) \cos \left[\omega_{c}\left(t-\tau_{1 n j}\right)\right] d t .
\end{gathered}
$$

Note that only the first term of (82) is the desired signal; the second term of (82) is the multiple access interference (MAI) produced by the same user from different transmit antennas; the third term of (82) is the MAI produced by other users; the fourth term of (82) is the AWGN. Using the Gaussian approximation in [30], (82) can be presented in the following form

$$
\hat{z}_{1 j}(t)=\sqrt{0.5 P_{1}} T_{s} \sum_{n=1}^{N} h_{1 n j} b_{n 1}+\eta_{1 j}
$$

where $\hat{z}_{1 j}(t)$ is the Gaussian random variable; $\eta_{1 j}$ is the combination of the interference and noise. The expected mean and variance of $\eta_{1 j}$ are given by

$$
\left\{\begin{array}{l}
E\left[\eta_{1 j}\right]=0 \\
\operatorname{Var}\left[\eta_{1 j}\right]=N(N-1) \frac{T_{s}^{2}}{6 G} \sum_{k=1}^{K} P_{k}+4 \sigma_{w}^{4} N T_{s}
\end{array}\right.
$$


respectively, where $G$ is the processing gain of the DS-CDMA system. Note that (83) has the same form as (4). After STBC decoding is performed on $\hat{z}_{1 j}(t)$ given in (83), the decision statistic for user

1 over $T$ symbol durations takes the form

$$
\hat{\mathbf{z}}_{1}(t)=R^{-1} \sqrt{0.5 P_{1}} T_{s}\|\mathbf{H}\|_{F}^{2} \mathbf{b}_{1 T}+\boldsymbol{\eta}_{T},
$$

where $\eta_{i T}(t)$ has the zero mean and the variance is defined as

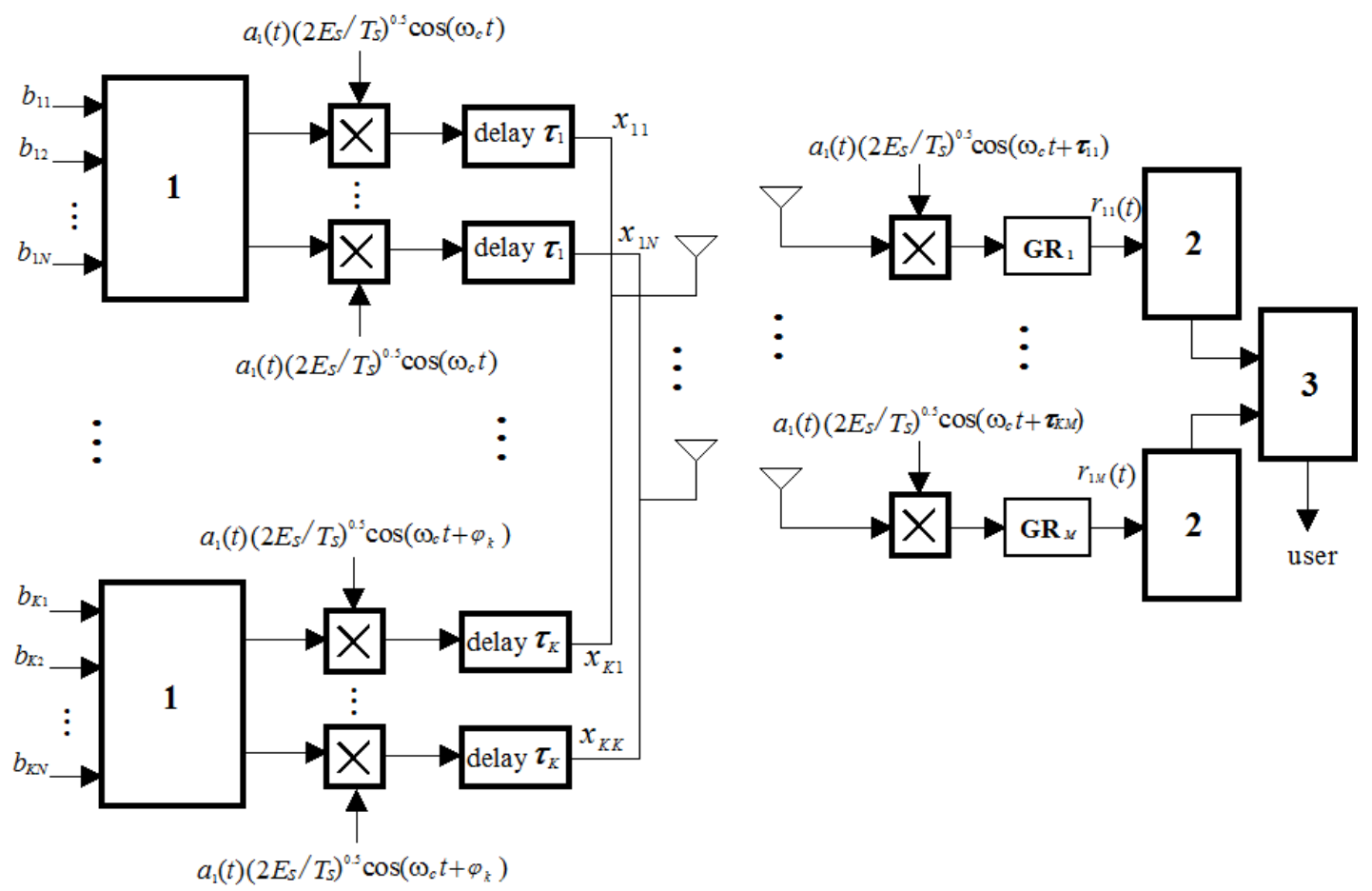

Fig. 2. System model for STBC DS-CDMA with multiple antennas employing the generalized receiver: 1 - STBC Encoder; 2 - STBC Decoder; 3 - Decision device: GR - Generalized receiver.

$\operatorname{Var}\left\{\eta_{i T}(t)\right\}=\frac{1}{R}\|\mathbf{H}\|_{F}^{2}\left[\frac{N(N-1) T_{s}^{2}}{6 G} \sum_{k=1}^{K} P_{k}+N T_{s} 4 \sigma_{w}^{4}\right]$.

Therefore, the effective instantaneous SNR at the generalized receiver output is given by

$$
\gamma_{s}=\left\{\frac{N(N-1) K}{3 G}+\frac{4 N \sigma_{w}^{4}}{E_{s}^{2}}\right\}^{-1} R^{-1}\|\mathbf{H}\|_{F}^{2} .
$$

Note that the perfect power control is assumed in (87), i.e., $P_{k}=P_{1}$.

\subsection{Capacity analysis of STBC-CDMA}

To facilitate the capacity analysis, we first normalize the equivalent channel by $\sqrt{0.5 T_{s}}$, then (85) can be written in the same form as (7)

$$
\mathbf{y}_{n T}=R^{-1}\|\mathbf{H}\|_{F}^{2} \mathbf{x}_{n T}+\boldsymbol{\eta}_{n T},
$$

where $\mathbf{x}_{n T}$ is the $S \times 1$ complex input matrix with each entry having symbol energy $E_{s}$; the combination of the interference and noise $\boldsymbol{\eta}_{n T}$ has the zero mean and variance

$$
\operatorname{Var}\left\{\boldsymbol{\eta}_{n T}\right\}=R^{-1} \frac{\|\mathbf{H}\|_{F}^{2} N(N-1) K}{3 G}+4 \sigma_{w}^{4} N .
$$

The capacity of this STBC CDMA system constructed based on the generalized approach to signal processing in noise [19]-[21] and employing a $q$-ary signal constellation can be obtained directly from (31).

\subsection{Probability of error for DS-CDMA with STBC}

The average $S N R$ per channel is determined as

$$
\begin{gathered}
\bar{\gamma}_{c}=\frac{E\left\{\left\|h_{i j}\right\|^{2}\right\}}{R\left\{\frac{N(N-1) K}{3 G}+\frac{4 N \sigma_{w}^{4}}{E_{s}^{2}}\right\}} \\
=\frac{2 \sigma^{2}}{R\left\{\frac{N(N-1) K}{3 G}+\frac{4 N \sigma_{w}^{4}}{E_{s}^{2}}\right\}}
\end{gathered}
$$

and this can be used with the probability of error results in Section 4 to obtain the performance in fad- 
ing channels. In particular, the exact BEP of BPSK is given by

$$
P_{S T B C_{-} \text {CDMA,Rayleigh }, 2 b}^{\text {erro }}=\frac{1}{2}\left[1-\sum_{k=0}^{M N-1} \mu\left(\frac{1-\mu^{2}}{4}\right)^{k}\left(\begin{array}{c}
2 k \\
k
\end{array}\right)\right]
$$

for the Rayleigh fading channel;

$$
\begin{array}{r}
P_{\text {STBC_CDMA,Rician }, 2 b}^{\text {error }}=\sum_{n=0}^{\infty} \frac{(M N \beta)^{n} \exp \{-M N \beta\}}{2 \Gamma(n+1)} \lambda \\
\times\left[1-\sum_{i=0}^{M N+n-1} \mu\left(\frac{1-\mu^{2}}{4}\right)^{i}\left(\begin{array}{c}
2 i \\
i
\end{array}\right)\right]
\end{array}
$$

for the Rician fading channel;

$$
P_{\text {STBC_CDMA,Naragami }, q}^{\text {error }}=\frac{\lambda}{2}\left[1-\sum_{k=0}^{m M N-1} \mu\left(\frac{1-\mu^{2}}{4}\right)^{k}\left(\begin{array}{c}
2 k \\
k
\end{array}\right)\right]
$$

for the Nakagami-m fading channel, where $\mu$ is given by (58) and $\bar{\gamma}_{c}$ is defined in (90). Note that there are two factors in $\bar{\gamma}_{c}$ which determine the BEP of the DS-CDMA wireless communication system with STBC, $N(N-1) K / 3 G$ and $4 \sigma_{w}^{4} N$. The first term corresponds to the MAI from other users and the selfinterference from different transmitting antennas. The second term corresponds to the system noise, i.e., AWGN. At the high $S N R, \bar{\gamma}_{c}$ will be dominated on the MAI, i.e., the number of users limits the performance, as expected.

\section{Numerical results}

In this section, some numerical results are presented to illustrate and verify the capacity and probability of error results obtained before. Figure 3 demonstrates the capacity using SBTC $\mathbf{G}_{2}$ over Rician fading channel with one, two and four receive antennas for BPSK, QPSK, and 8-PSK. The Rician parameter is $\beta=100$. This figure shows that the capacity with STBC $_{2}$ is not increased as the number of receiving antennas increases. However, the SNR threshold required to achieve capacity improves as the number of receive antennas increased. The capacity with a single antenna over Rician fading channel for PSK is included for reference. Additionally, Fig. 3 represents a superiority of employment of the generalized receiver in DS-CDMA wireless communication system in comparison with the correlation one.

The capacity using several STBCs over Rayleigh fading channel is presented in Fig.4. As expected, it shows that $\mathbf{G}_{2}$ is the optimal code from a cap acity perspective, and $\mathbf{H}_{4}$ is more efficient in comparison with $\mathbf{G}_{4}$. Also, a demonstration of superiority to em- ploy the generalized receiver in comparison with the correlation one in DS-CDMA wireless communication system with STBCs is demonstrated in Fig.4. Comparison between Figs. 3 and 4 demonstrates that the DS-CDMA wireless communication system with STBC codes achieves the capacity at the lower

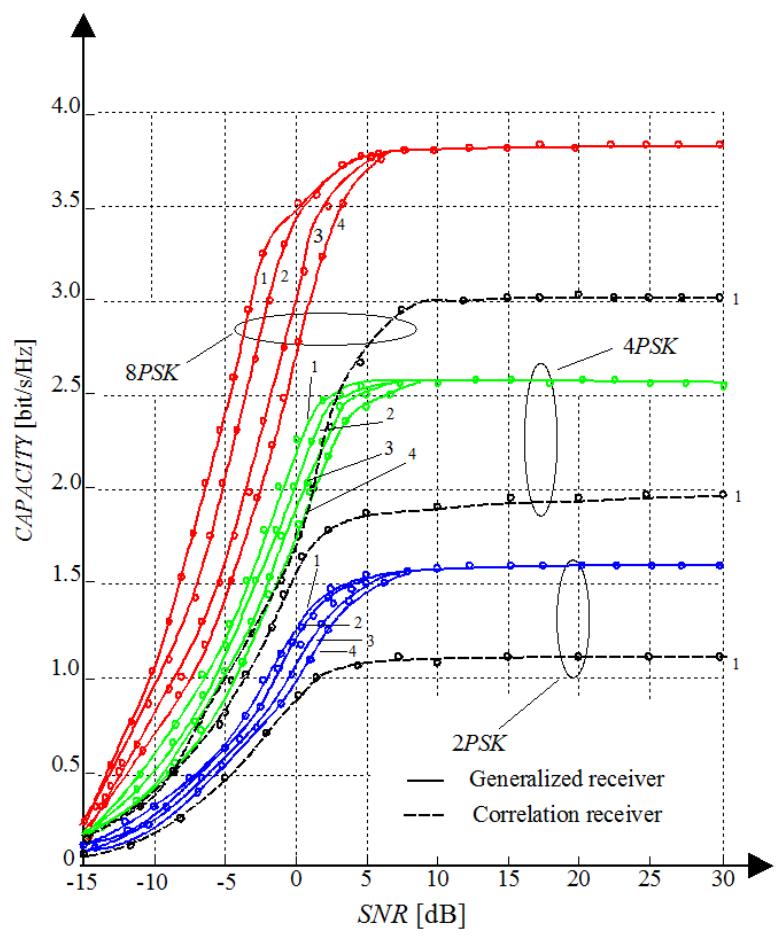

Fig. 3. Capacity of DS-CDMA system employing STBC $\mathbf{G}_{2}$ over Rician fading channel with Rician parameter $\beta=100$ : 1 - MIMO 4x2; 2 - MIMO 2x2; 3 - MIMO $1 \times 2 ; 4-$ MIMO $1 \times 1$.

SNR over the Rician fading channel at $\beta=100$ than the Rayleigh fading channel. Figure 5 demonstrates the relationship between the Rician parameter $\beta$ and the capacity if STBC code $\mathbf{G}_{2}$ is used. Note that the capacity is insensitive to the Rician parameter when $\beta>15 \mathrm{~dB}$. Additionally, we can see a superiority of implementation of the generalized receiver over the correlation one in DS-CDMA wireless communication system with STBC codes.

Simulation was used to verify the exact and approximate error probabilities given in Section 4. In Fig.6, the BEP of QPSK at STBCs $\mathbf{G}_{2}, \mathbf{G}_{3}, \mathbf{G}_{4}, \mathbf{H}_{3}$ and $\mathbf{H}_{4}$ with one and two receive antennas are presented, and these results are identical to those obtained using (48). Also, a superiority of employment of the generalized receiver in DS-CDMA system with STBC in comparison with the correlation one is evident. Figure 7 shows the comparison between the approximate and exact (via simulation) SEP for 8PSK. Note that the approximation error is negligible 


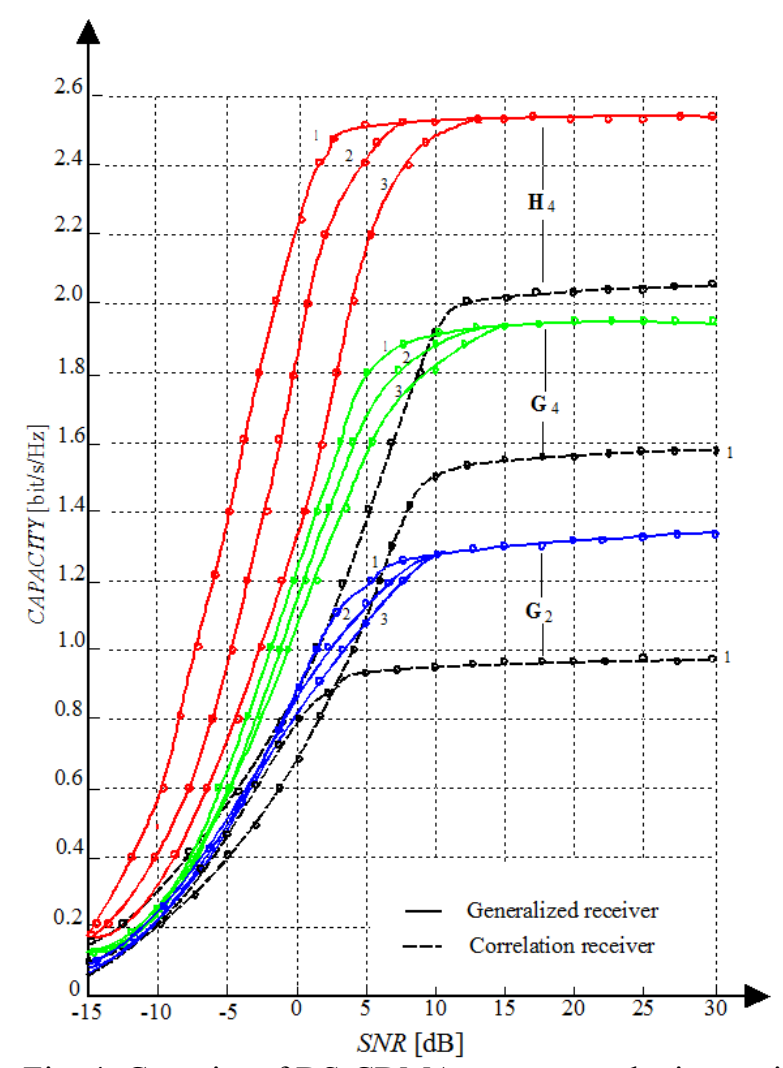

Fig. 4. Capacity of DS-CDMA system employing various STBCs over Rayleigh fading channel: 1 - MIMO 4x2; 2 - MIMO 2x2; 3 - MIMO 1x2.

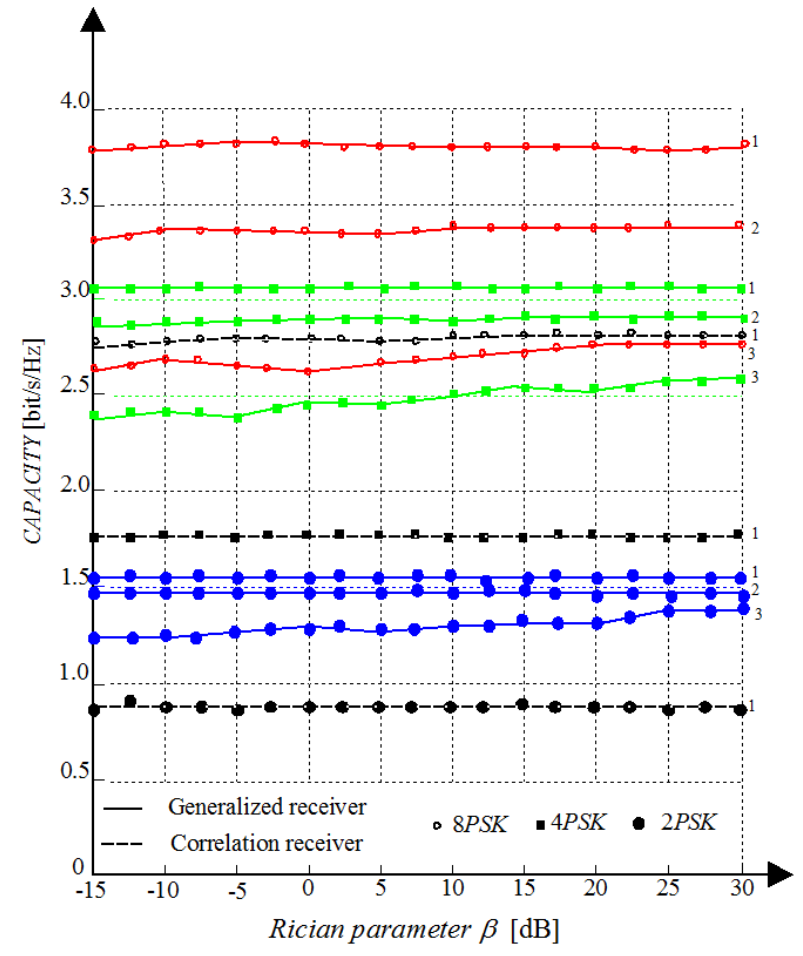

Fig. 5. Capacity of DS-CDMA system with STBC $\mathbf{G}_{2}$ versus the Rician parameter $\beta$; SNR $=5 \mathrm{~dB}: 1-$ MIMO $4 \times 2 ; 2$ - MIMO 2x2; 3 - MIMO 1x2.

Figure 8 demonstrates the SEP for the 16-QAM with one and two receive antennas for different
STBCs. A superiority of implementation of the generalized receiver in DS-CDMA system with STBC over the correlation one is presented, too. These results are identical to those obtained with (39).

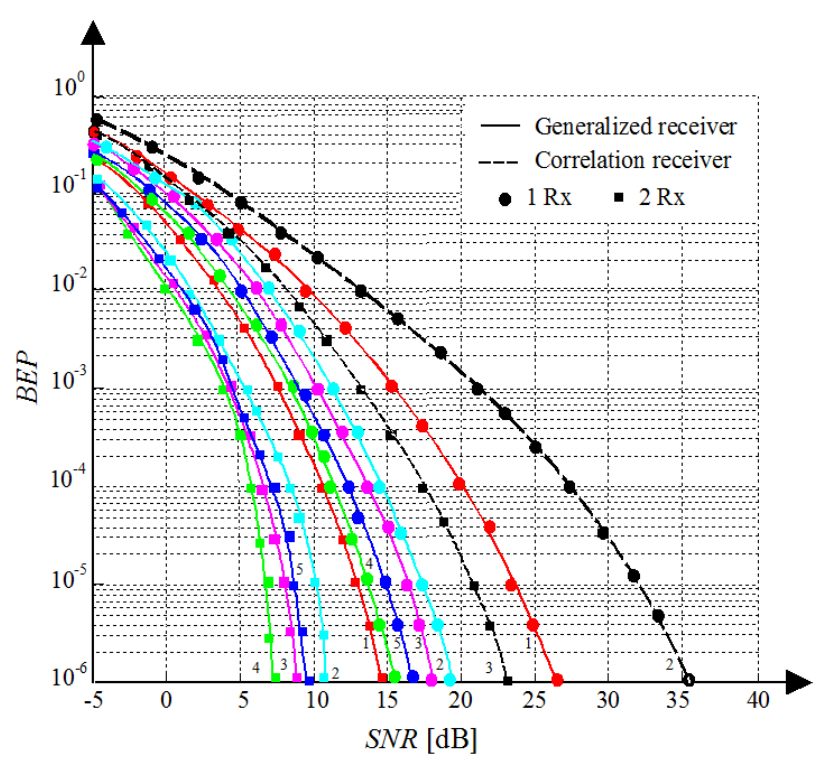

Fig. 6. BEP of DS-CDMA system with QPSK for STBC using one and two receive antennas over Rayleigh fading channel:1 - STBC $\mathbf{G}_{2} ; 2-\mathrm{STBC} \mathbf{G}_{3} ; 3-\mathrm{STBC} \mathbf{H}_{3} ; 4$ $-\mathrm{STBC} \mathbf{G}_{4} ; 5-\mathrm{STBC} \mathbf{H}_{4}$.

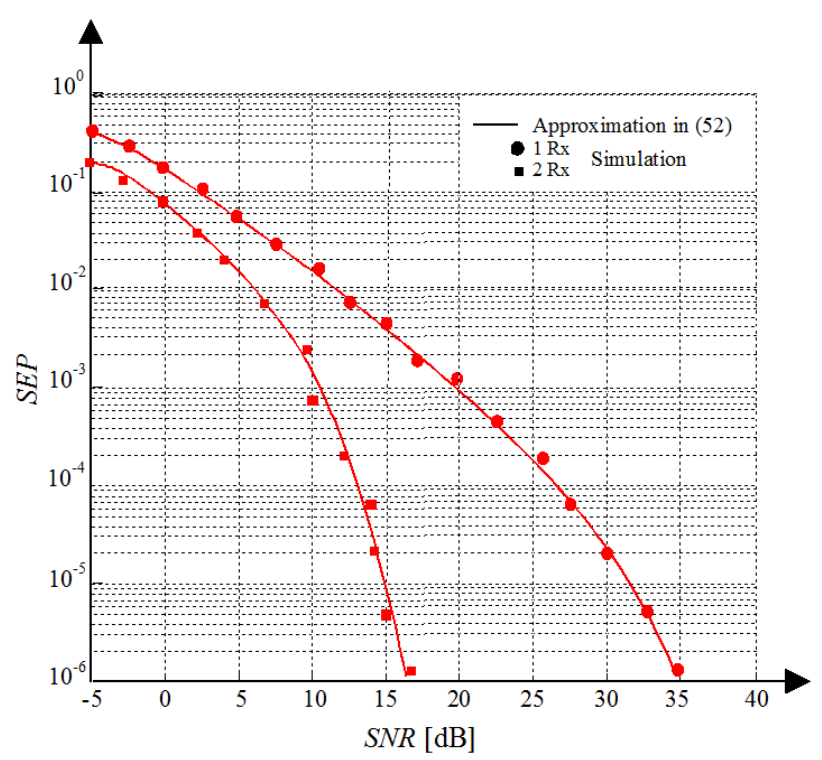

Fig. 7. SEP of DS-CDMA system with QPSK for STBC using one and two receive antennas over Rayleigh fading channel:1 - STBC $\mathbf{G}_{2} ; 2-\mathrm{STBC} \mathbf{G}_{3} ; 3-\mathrm{STBC} \mathbf{H}_{3} ; 4$ $-\mathrm{STBC} \mathbf{G}_{4} ; 5-\mathrm{STBC} \mathbf{H}_{4}$.

In Fig.9 the capacity of DS-CDMA wireless communication system based on the generalized approach to signal processing in noise [19]-[21] with several STBCs is demonstrated for the case of BPSK modulation over the Rayleigh fading channel. 
We can see that with a given number of users and signal processing gain, the system may not be able to achieve the full channel capacity even with infinite

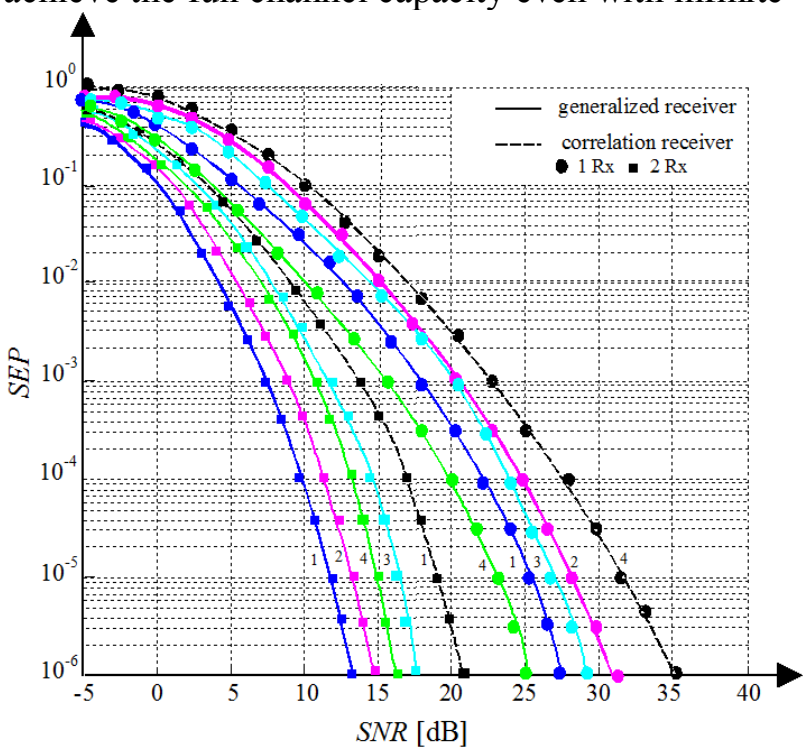

Fig. 8. SEP of DS-CDMA system with 16-QAM for STBC using one and two receive antennas over Rayleigh fading channel: 1 - STBC $\mathbf{H}_{4} ; 2-$ STBC $\mathbf{H}_{3} ; 3-$ STBC $\mathbf{G}_{3} ; 4-\mathrm{STBC}_{\mathbf{G}}$.

SNR due to the dominant MAI component. In this case, increasing the number of antennas will increase the achievable DS-CDMA wireless communication system capacity. Note that the DS-CDMA wireless communication system capacity increases significantly as the number of receive antennas increases for a given number of users and signal processing gain, as expected. However, it should be nted that if the DS-CDMA wireless communication system can already achieve the channel capacity for a given number of antennas, users and signal processing gain, increasing the number of antennas cannot increase the DS-CDMA wireless communication system capacity.

Figure 10 presents us the relationship between the DS-CDMA wireless communication system capacity and the number of users given the processing gain and SNR over the Rayleigh fading channel. It can be seen that the capacity decreases rapidly as the number of users is increased for one and two receive antennas. However, with four receive antennas increasing the number of users has much less effect on the DS-CDMA wireless communication capacity.

The BEP for DS-CDMA wireless communication system constructed on the basis of the generalized approach to signal processing in noise with STBC using BPSK modulated signals is presented in Fig. 11 for one, two, three, and four receive antennas. The signal processing gain is 64 , the number of us-

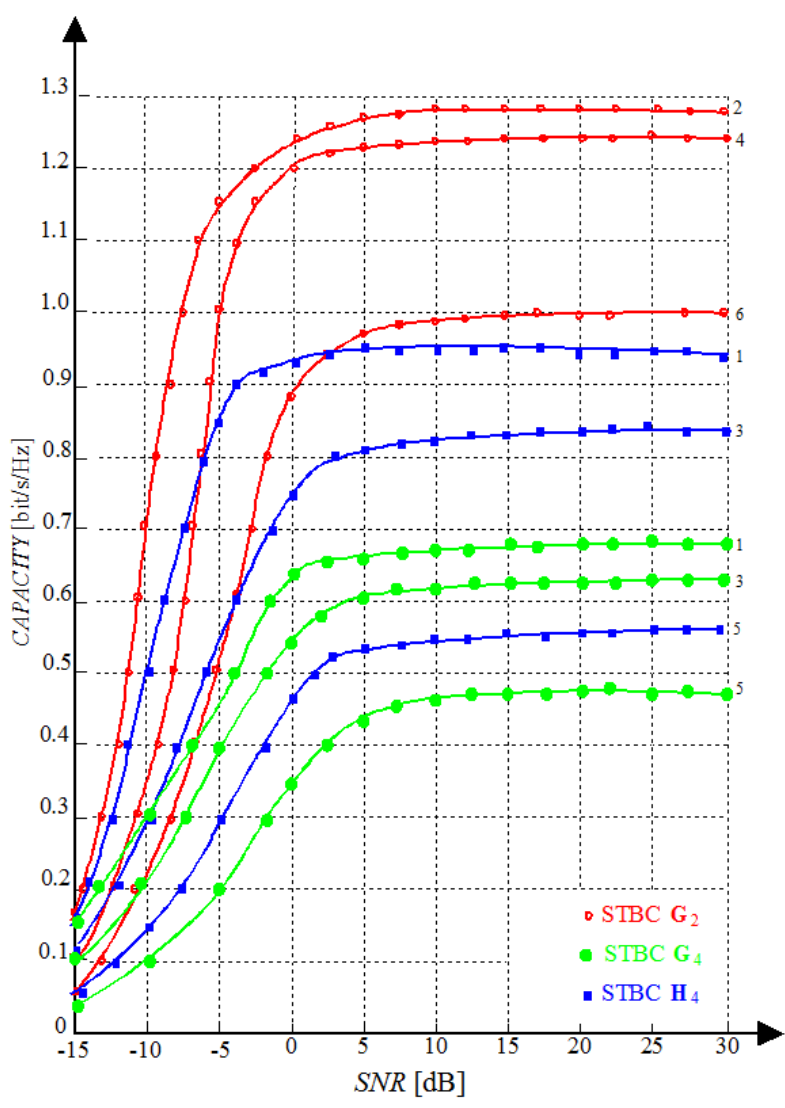

Fig. 9. Capacity of DS-CDMA system with BPSK, STBC over Rayleigh fading channel $G=32, K=30: 1$ - MIMO $4 \times 4 ; 2$ - MIMO 4x2; 3 - MIMO 2x4; 4- MIMO 2x2; 5 MIMO 1x4; 6- MIMO 1x2.

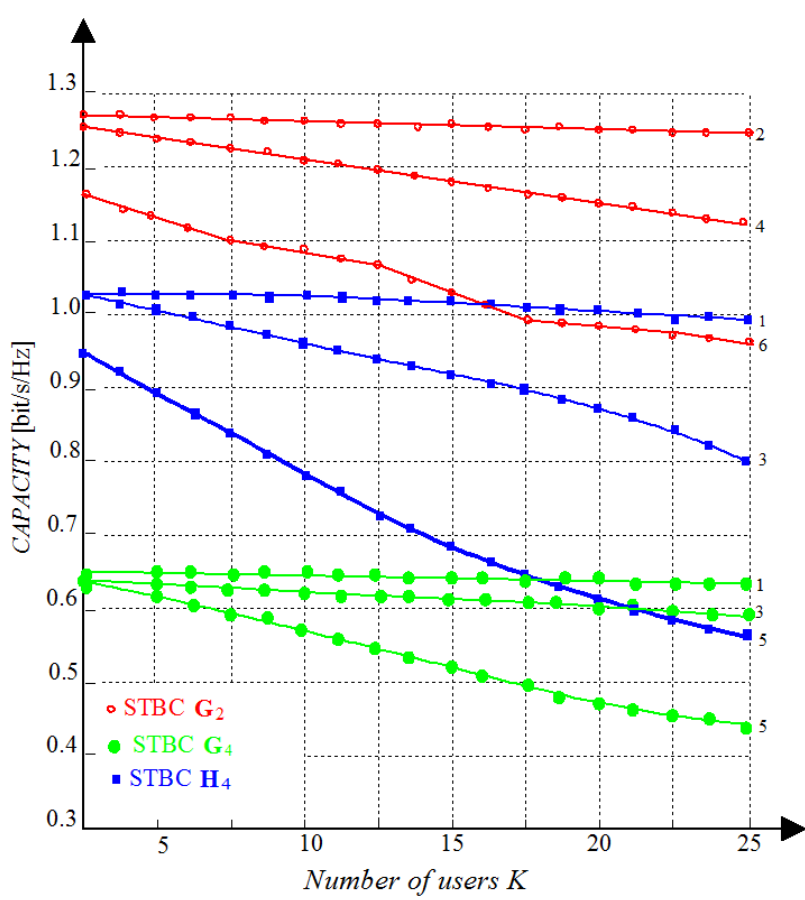

Fig. 10. Capacity of DS-CDMA system with BPSK and STBC over Rayleigh fading channel $G=32, S N R=7 \mathrm{~dB}: 1$ - MIMO 4x4; 2 - MIMO 4x2; 3 - MIMO 2x4; 4- MIMO $2 \times 2 ; 5$ - MIMO 1x4;6-MIMO 1×2. 
ers is 20 and the Rayleigh fading is employed for all DS-CDMA wireless communication system figures. Figure 11 presents that significant performance gain

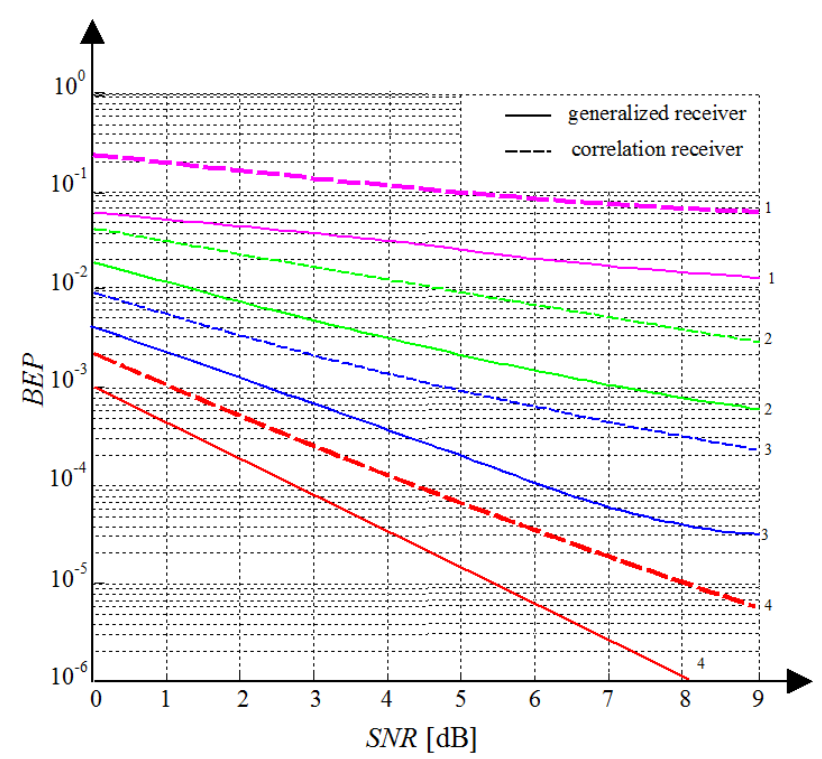

Fig. 11. BEP of DS-CDMA system with BPSK and STBC $\mathbf{G}_{2}$ over Rayleigh fading channel $G=64, K=20: 1$ - one receive antenna; 2 - two receive antennas; 3 - three receive antennas; 4 - four receive antennas.

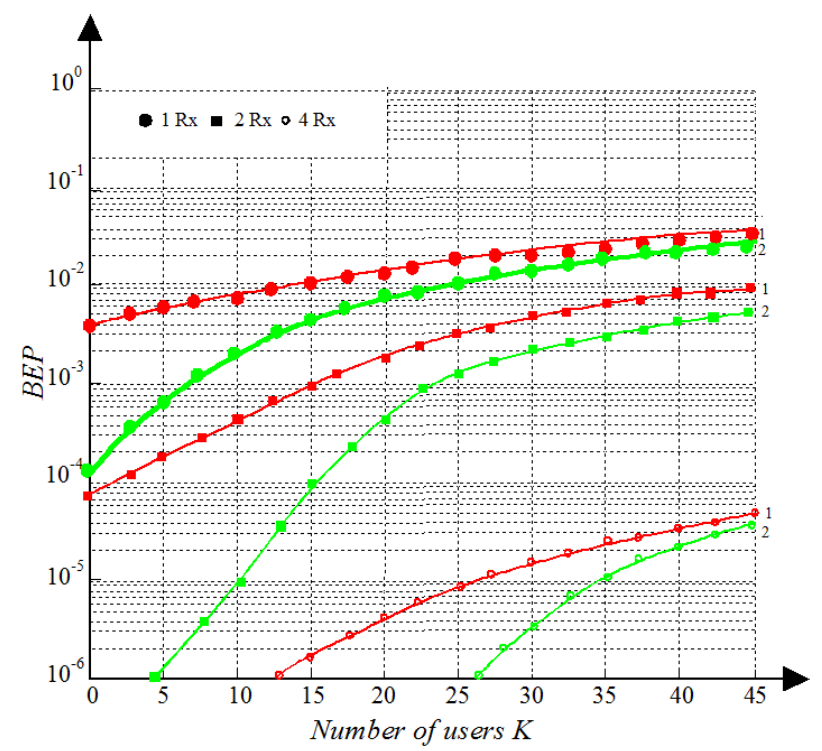

Fig. 12. BEP versus the number of users for DS-CDMA system with BPSK, STBC $\mathbf{G}_{2}$ (the curve 1) and $\mathbf{G}_{4}$ (the curve 2) over the Rayleigh fading channel with $G=64$, $S N R=7 \mathrm{~dB}$.

can be obtained with multiple receive antennas. For comparison the simulation results for the correlation receiver are presented, too. We can see a great superiority of implementation of the generalized receiver in DS-CDMA wireless communication system with STBC over the correlation one.
Figure 12 demonstrates the relationship between the $B E P$ and the number of users in the DS-CDMA wireless communication system at SNR equal to 7 $\mathrm{dB}$ with $\mathbf{G}_{2}$ and $\mathbf{G}_{4}$. As the number of users is increased, the performance degrades, but at the four receive antennas the DS-CDMA wireless communication system is capable of accommodating far more users than with one receive antenna. As it is shown in the Fig. 12, $\mathbf{G}_{4}$ provides the better performance than $\mathbf{G}_{2}$, however, this is obtained at the price of the capacity loss as shown in Fig. 10.

\section{Conclusions}

The capacity and error probability of STBCs employed by DS-CDMA wireless communication system with STBC constructed on the basis of the generalized approach to signal processing in noise have been studied in fading channels for $q$-ary signal constellations. The Rayleigh, Rician, and Nakagami-m fading channels were investigated for the PAM, PSK, and QAM modulations. The closed form of the error probabilities under employment of the generalized receiver based on the generalized approach to signal processing in noise were derived for various fading and modulation combinations. This analysis has been employed to determine the performance of STBC multiuser DS-CDMA wireless communication system. Comparative analysis concerning implementation of the generalized receiver and correlation one in DS-CDMA wireless communication system with STBCs demonstrates a great superiority in favor of the first.

\section{Appendix 1}

Using integration by parts, the proof of (41) is as follows:

$$
\begin{gathered}
g(L)=\int_{0}^{\infty} Q(\sqrt{a x}) x^{L-1} \exp \{-x / u\} d x \\
=-\int_{0}^{\infty} Q(\sqrt{a x}) x^{L-1} u d\{\exp [-x / u]\} \\
=-Q(\sqrt{a x}) x^{L-1} u \exp \{-x / u\} \\
+\int_{0}^{\infty} \exp \{-x / u\} u\left[Q(\sqrt{a x}) x^{L-1}\right] d x \\
=u(L-1) \int_{0}^{\infty} \exp \{-x / u\} Q(\sqrt{a x}) x^{L-2} d x \\
-u \sqrt{a / 8 \pi} \int_{0}^{\infty} x^{L-3 / 2} \exp \{-x / u\} \exp \{-a x / 2\} d x \\
=u(L-1) g(L-1)-\frac{1}{2} u^{L} \mu\left(\frac{1-\mu^{2}}{4}\right)^{L-1} \frac{(2 L-2) !}{(L-1) !},
\end{gathered}
$$


where

$$
\mu=\sqrt{\frac{a u}{2+a u}} .
$$

Repeating the process, we have

$$
g(L)=u^{L-1} \Gamma(L) g(1)-\frac{1}{2} u^{L} \Gamma(L) \sum_{k=2}^{L} \mu\left(\frac{1-\mu^{2}}{4}\right)^{k-1}\left(\begin{array}{c}
2 k-2 \\
k-1
\end{array}\right)
$$

Now, using the fact that

$$
g(1)=\int_{0}^{\infty} Q(\sqrt{a x}) \exp \{-x / u\} d x=\frac{1}{2} u(1-\mu),
$$

we obtain (41)

$$
g(L)=\frac{1}{2} u^{L} \Gamma(L)\left[1-\sum_{k=0}^{L-1} \mu\left(\frac{1-\mu^{2}}{4}\right)^{k}\left(\begin{array}{c}
2 k \\
k
\end{array}\right)\right] .
$$

\section{Appendix 2}

The truncation of (68) to the first $L$ terms will introduce the error

$$
\begin{gathered}
\text { Error }=\lambda \sum_{i=L}^{\infty} \mu\left(\frac{1-\mu^{2}}{4}\right)^{M N+i}\left(\begin{array}{c}
2(M N+i \\
M N+i
\end{array}\right) \\
\times\left[1-\sum_{n=0}^{i} \frac{(M N K)^{n} \exp \{-M N K\}}{\Gamma(n+1)}\right] .
\end{gathered}
$$

Given the fact that

$$
4^{-M N-i}\left(\begin{array}{c}
2(M N+i) \\
M N+i
\end{array}\right)<1
$$

for all $i$, we have

$$
\begin{gathered}
\lambda \sum_{i=L}^{\infty} \mu\left(\frac{1-\mu^{2}}{4}\right)^{M N+i}\left(\begin{array}{c}
2(M N+i) \\
M N+i
\end{array}\right) \\
\times\left[1-\sum_{n=0}^{i} \frac{(M N K)^{n} \exp \{-M N K\}}{\Gamma(n+1)}\right]<\lambda \mu \sum_{i=L}^{\infty}\left(1-\mu^{2}\right)^{M N+i} \\
\times\left[1-\sum_{n=0}^{L} \frac{(M N K)^{n} \exp \{-M N K\}}{\Gamma(n+1)}\right]
\end{gathered}
$$

The right-hand side of (94) can be simplified to

$$
\begin{gathered}
\lambda \mu \sum_{i=L}^{\infty}\left(1-\mu^{2}\right)^{M N+i}\left[1-\sum_{n=0}^{L} \frac{(M N K)^{n} \exp \{-M N K\}}{\Gamma(n+1)}\right] \\
=\lambda \mu\left[1-\sum_{n=0}^{L} \frac{(M N K)^{n} \exp \{-M N K\}}{\Gamma(n+1)}\right] \\
\left(1-\mu^{2}\right)^{M N+L} \sum_{i=0}^{\infty}\left(1-\mu^{2}\right)^{i}
\end{gathered}
$$

Given that

$$
\sum_{i=0}^{\infty} q^{i}=\frac{1}{1-q},-1 \leq q \leq 1,
$$

(94) can be written as

$$
\begin{gathered}
\lambda \sum_{i=L}^{\infty} \mu\left(\frac{1-\mu^{2}}{4}\right)^{M N+i}\left(\begin{array}{c}
2(M N+i) \\
M N+i
\end{array}\right) \\
\times\left[1-\sum_{n=0}^{i} \frac{(M N K)^{n} \exp \{-M N K\}}{\Gamma(n+1)}\right]<\lambda \frac{\left(1-\mu^{2}\right)^{M N+L}}{\mu} \\
\times\left[1-\sum_{n=0}^{L} \frac{(M N K)^{n} \exp \{-M N K\}}{\Gamma(n+1)}\right] \cdot
\end{gathered}
$$

Thus, truncation of (68) to the first $N$ terms will introduce an error

$$
\text { Error }=\lambda\left[1-\sum_{n=0}^{L} \frac{(M N K)^{n} \exp \{-M N K\}}{\Gamma(n+1)}\right]\left(1-\mu^{2}\right)^{M N+L}
$$

in the error probability.

\section{References:}

[1] Alamouti, S.M. A simple transmit diversity technique for wireless communications. IEEE Journal of Selection Areas on Communications.1998, Vol. 16, No. 8, pp. 1452-1458.

[2] Tarokh,V., Jafarkhani,H., Claderbank, A.R. Space-time block codes from orthogonal designs. IEEE Transactions on Information Theory.1999, Vol. 45, No. 5, pp. 1456-1467.

[3] Tarokh,V. Space-time block coding for wireless communications: Performance results. IEEE JOurnal of Selection Areas on Communications. 1999, Vol. 16, No. 8, pp. 1452-1458.

[4] Sandhu, S., Paulraj, A. Space-time block codes: A capacity perspective. IEEE Communications Letters. 2000, Vol.4, No.12, pp. 384-386.

[5] Yue, D.W., Wang, Q. Capacity of orthogonal space-time block codes in MISO fading channels with co-channel interferences and noise. Science in China. Series F: Information Sciences. 2009, Vol. 52, pp. 1697-1703.

[6] Dai, L., Sfar, S., Lefaief, K.B. Optimal antenna selection based on capacity maximization for MIMO systems in correlated channels. IEEE Transactions on Communications. 2006, Vol. 54, No. 3, pp. 563-573.

[7] Proakis, J.G., Salehi, M. Digital Communications. $5^{\text {th }}$ Edition. New York: McGraw-Hill, USA. 2007, 1150 pages.

[8] Li, X., Luo, T., Yue, G., Yin, C. A squaring method to simplify the decoding of orthogonal space-time block codes. IEEE Transactions on Communications. 2001, Vol. 49, No.10, pp. 17001703.

[9] Taricco, G., Biglieri, E. Exact pair wise error probability of space-time codes. IEEE Transactions on Information Theory. 2002, Vol. 48, No. 2, pp. 510-514. 
[10] Kshetrimayum, R. Advanced topics in MIMO wireless communications. Chapter in Fundamentals of MIMO Wireless Communications. Cambridge: Cambridge University Press. 2017, pp. 270-308.

[11] Martins, C., Brandao,M.L.,Brandani da Silva, E. New space-time block codes from spectral norm. PLoS ONE. 2019, Vol. 14, No.9: e02227 083.https://doi.org/10.371/journal.pone.222708

[12] Alouini, M.-S., Goldsmith, A.J. A unified approach for calculating error rates of linearly-modulated signals over generalized fading channels IEEE Transactions on Communications. 1999, Vol. 47, No. 9, pp. 1324-1334.

[13] Foschini, G.J., Gans, M. On limits of wireless communications in a fading environment when using multiple antennas. Wireless Personal Communications.1998, Vol. 6, pp. 311-335.

[14] Ungerboeck, G. Channel coding with multiplephase signals. IEEE Transactions on Information Theory.1982, Vol. 28, No. 1, pp. 55-67.

[15] Baccarelli, E., Fasano, A. Some simple bounds on the symmetric capacity and outage probability for QAM wireless channels with Rice and Nakagami fading," IEEE Journal of Selected Areas on Communications. 2000, Vol. 18, No. 3, pp. 361-368.

[16] Baccarelli, E. Evaluation of the reliable data rates supported by multiple-antenna coded wireless links for QAM transmissions. IEEE Journal of Selected Areas on Communications. 2001, Vol. 19, No. 2, pp. 295-304.

[17] Proakis, G.J. Probabilities of error for adaptive reception of M-phase signals," IEEE Transactions on Communication Technology.1968, Vol. 16, No. 1, pp. 71-81.

[18] Shayesteh, M., Aghamohammadi, A. On the error probability of linearly modulated signals on frequency-flat Rician, Rayleigh, and AWGN channels. IEEE Transactions on Communications. 1995, Vol. 43, No. 2, pp. 1454-1466.

[19] Tuzlukov, V.P. A new approach to signal detection theory. Digital Signal Processing. 1998, Vol. 8, No. 3, pp. 166-184.

[20] Tuzlukov, V.P. Signal Detection Theory, New York: Springer-Verlag, 2001.

[21] Tuzlukov, V.P. Signal Processing Noise, Boca Raton, London, New York, Washington D.C.: CRC Press, Taylor \& Francis Group, 2002.

[22] Maximov, M. Joint correlation of fluctuative noise at outputs of frequency filters. Radio Engineering. 1956, No. 9, pp. 28-38.

[23] Chernyak, Y. Joint correlation of noise voltage at outputs of amplifiers with no overlapping responses. Radio Physics and Electronics. 1960,
No. 4, pp. 551-561.

[24] Tuzlukov, V.P. DS-CDMA downlink systems with fading channel employing the generalized. Digital Signal Processing. 2011. Vol. 21, No. 6, pp. 725-733.

[25] Tuzlukov, V.P. Signal processing by generalized detector in DS-CDMA wireless communication systems with frequency-selective channels. Circuits, Systems, and Signal Processing. Published on-line on February 2, 2011, doi:10. 1007/s00034-011-9273-1; 2011, Vol.30, No.6, pp. 1197-1230.

[26] Shbat, M., Tuzlukov, V.P. Primary signal detection algorithms for spectrum sensing at low SNR over fading channels in cognitive radio. Digital Signal Processing (2019). https://doi. org/10.1016/j.dsp. 2019.07.16. Digital Signal Processing. 2019, Vol. 93, No. 5, pp. 187- 207.

[27] Shbat, M., Tuzlukov, V.P. Evaluation of detection performance under employment of the generalized detector in radar sensor systems. $R a-$ dioengineering. 2014, Vol. 23, No. 1, pp. 5065.

[28] Shbat, M., Tuzlukov, V.P. Definition of adaptive detection threshold under employment of the generalized detector in radar sensor systems. IET Signal Processing. 2014, Vol. 8, Issue 6, pp. 622-632.

[29] Shbat, M., Tuzlukov,V.P. SNR wall effect alleviation by generalized detector employment in cognitive radio networks. Sensors, 2015, 15(7), pp.16105-16135;doi:10.3390/ s150716105.

[30] Wei, S. X. An alternative derivation for the signal-to-noise ratio of SSMA system. IEEE Transactions on Communications. 1994, Vol. 42, pp. 2224-2226

[31] Rappaport, T.S. Wireless Communications:Principles and Practice. Englewood Cliffs, NJ: Prentice-Hall, 1996.

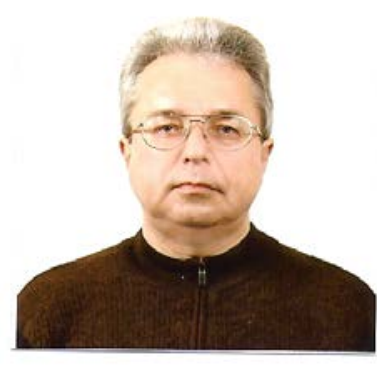

Dr. Vyacheslav Tuzlukov received the $\mathrm{MSc}$ and $\mathrm{PhD}$ degrees in radio physics from the Belarussian State University, Minsk, Belarus in 1976 and 1990, respectively, and DSc degree in radio physics from the Kotelnikov Institute of Radioengineering and Electronics of Russian Academy of Sciences in 1995. Starting from 1995 and till 1998 Dr. Tuzlukov was a Visiting Professor at the University of San-Diego, San-Die- 
go, California, USA. In 1998 Dr. Tuzlukov relocated to Adelaide, South Australia, where he served as a Visiting Professor at the University of Adelaide till 2000. F rom 2000 to 2002 he was a Visiting Professor at the University of Aizu, Aizu-Wakamatsu City, Fukushima, Japan and from 2003 to 2007 s erved as an Invited Professor at the Ajou University, Suwon, South Korea, within the Department of Electrical and Computer Engineering. Starting from March 2008 to February 2009 he joined as a Full Professor at the Yeungnam University, Gyeonsang, South Korea within the School of Electronic Engineering, Communication Engineering, and Computer Science. Starting from March 1, 2009 Dr. Tuzlukov served as Full Professor and Director of Signal Processing Lab at the Department of Communication and Information Technologies, School of Electronics Engineering, College of IT Engineering, Kyungpook National University, Daegu, South Korea. Currently, Dr. Tuzlukov is the Head of Department of Technical Exploitation of Aviation and Radio Engineering Equipment, Belarussian State Academy of Aviation, Minsk, Belarus. His research emphasis is on signal processing in radar, wireless communications, wireless sensor networks, remote sensing, sonar, satellite communications, mobile communications, and other signal processing systems. He is the author over 280 journal and conference papers, seventeenth books in signal processing published by Springer-Verlag and CRC Press. Some of them are Signal Detection Theory (2001), Signal Processing Noise (2002), Signal and Image Processing in Navigational Systems (2005), Signal Processing in Radar Systems (2012), Editor of the book Communication Systems: New Research (2013), Nova Science Publishers, Inc, USA, and has also contributed Chapters "Underwater Acoustical Signal Processing" and "Satellite Communications Systems: Applications" to Electrical Engineering Handbook: $3^{\text {rd }}$ Edition, 2005, CRC Press; "Generalized Approach to Signal Processing in Wireless Communications: The Main Aspects and Some Examples" to Wireless Communications and Networks: Recent Advances, InTech, 2012; "Radar Sensor Detectors for Vehicle Safety Systems" to Electrical and Hybrid Vehicles: Advanced Systems, Automotive Technologies, and Environmental and Social Implications, Nova Science Publishers, Inc., USA, 2014; "Wireless Communications: Generalized Approach to Signal Processing" and "Radio Resource Management and Femtocell Employment in LTE Networks", to Communication Systems: New Research, Nova Science Publishers, Inc., USA, 2013, and "Radar Sensor Detect- ors for Vehicle Safety Systems" to Autonomous Vehicles: Intelligent Transport Systems and Automotive Technologies, Publishing House, University of Pitesti, Romania, 2013. He participates as the General Chair, Keynote Speaker, Plenary Lecturer, Chair of Sessions, Tutorial Instructor and organizes Special Sections at the major International Conferences and Symposia on signal processing.

Dr. Tuzlukov was highly recommended by U.S. experts of Defence Research and Engineering (DDR\& E) of the United States Department of Defence as a recognized expert in the field of humanitarian demining and minefield sensing technologies and had been awarded by Special Prize of the United States Department of Defence in 1999 Dr. Tuzlukov is distinguished as one of the leading achievers from around the world by Marquis Who's Who and his name and biography have been included in the Who's Who in the World, 2006-2013; Who's Who in World, $25^{\text {th }}$ Silver Anniversary Edition, 2008, Marquis Publisher, NJ, USA; Who's Who in Science and Engineering, 2006-2012 and Who's Who in Science and Engineering, 10th Anniversary Edition, 2008-2009, Marquis Publisher, NJ, USA; 2009-2010 Princeton Premier Business Leaders and Professionals Honours Edition, Princeton Premier Publisher, NY, USA; 2009 Strathmore's Who's Who Edition, Strathmore's Who's Who Publisher, NY, USA; 2009 Presidental Who's Who Edition, Presidental Who's Who Publisher, NY, USA; Who's Who among Executives and Professionals, 2010 Edition, Marquis Publisher, NJ, USA; Who's Who in Asia 2012, $2^{\text {nd }}$ Edition, Marquis Publisher, NJ, USA; Top 100 Executives of 2013 Magazine, Super Network Publisher, New York, USA, 2013; 2013/2014 Edition of the Global Professional Network, Business Network Publisher, New York, USA, 2013; 2013/2014 Edition of the Who's Who Network Online, Business Network Publisher, New York, USA, 2014; Online Professional Gateway, 2014 Edition, Business Network Publisher, New York, USA, 2014; 2014 Worldwide Who's Who", Marquis Publisher, NJ, USA; 2015 Strathmore Professional Biographies, Strathmore's Who's Who Publisher, NY, USA; Who's Who in World, 2015, Marquis Publisher, NJ, USA; 2015-2016 Membership in Exclusive Top 100 network of professionals in the world, NY, USA, 2015; 2015 Who's Who of Executives and Professionals Honors Edition, Marquis Publisher, NJ, USA; Worldwide Who's Who - Top 100 Business Networking, San Diego, CA, USA, 2015.

Phone: +375173453283

Email: slava.tuzlukov@,mail.ru

\section{Creative Commons Attribution License 4.0 (Attribution 4.0 International, CC BY 4.0)}

This article is published under the terms of the Creative Commons Attribution License 4.0

https://creativecommons.org/licenses/by/4.0/deed.en US 